\title{
Model Predictive Control Guidance with Extended Command Governor Inner-Loop Flight Control for Hypersonic Vehicles
}

\author{
Christopher Petersen, Morgan Baldwin $\rceil^{*}$ and Ilya Kolmanovsky
}

\begin{abstract}
The paper describes a control system for hypersonic vehicles that consists of an outerloop guidance layer and an inner-loop flight control layer. For the outer-loop, a Model Predictive Control approach is pursued to prescribe the desired bank angle and flight path angle commands so that the vehicle can follow the way points and avoid exclusion zones during its flight. For the inner-loop, a combination of a Linear Quadratic state feedback control and an Extended Command Governor to handle pointwise-in-time state and control constraints is proposed. Simulation results are presented for an implementation of the proposed approach that includes the outer-loop MPC bank angle/flight path angle control and the inner-loop controller that tracks the desired angle-of-attack and enforces the constraints.
\end{abstract}

\section{Introduction}

Hypersonic flight is an area of great interest, with several experimental vehicles having been built and launched in recent years. Two test vehicles were flown under DARPA's Falcon Project, but both flights ended unsuccessfully during the glide phase. The Falcon program concluded that there were two major problems; the first being that the assumptions of known flight regimes were inadequate for flight, and the second being that the advanced thermal modeling and ground testing were not successful at predicting reality [1]. Hence it was found that conventional control techniques appropriate for subsonic and supersonic flight are not necessarily applicable for hypersonic flight.

Therefore, a control method for hypersonic vehicles that can handle model uncertainties and perturbations while adhering to strict flight constraints is necessary. One such solution is by utilizing predictive control. Two predictive controllers were made in [2]; the first was designed to track the velocity setpoint of the vehicle and the second, based on multi-model switching, was implemented to extend the approach to a larger flight envelope. In [3], an acceptable closed-loop performance for hypersonics was attained while satisfying hard control and fuel constraints. This was completed by using a generalized predictive controller with a quadratic cost function and a receding, finite prediction horizon. A more specific predictive approach to hypersonic control utilizes Model Predictive Control (MPC), where a robust controller is designed given constraints on the inputs and outputs of the system. A linear parameter-varying model with an MPC controller that considers actuator saturation was detailed in [4]. The topic of MPC for the robust control of a hypersonic fly vehicle has also been explored in [5].

In order to guarantee constraint enforcement during flight, reference governors for hypersonic gliders have also been developed. In particular, a reference governor augmented to an existing adaptive controller to track reference signals was implemented in [6] to avoid the occurrence of actuator saturation and prevent windup. A reference governor was also used in [7] to enforce constraints on elevator deflection for conventional UAV gliders.

In this work, a two-layered guidance and control system for hypersonic vehicles is considered, as shown in Fig. 1. In this framework, the outer-loop determines a reference flight path angle $\gamma_{\text {des }}$ and a reference

\footnotetext{
*Graduate Student, Department of Aerospace Engineering, The University of Michigan, Ann Arbor, MI.

${ }^{\dagger}$ Research Aerospace Engineer, Space Vehicles Directorate, Air Force Research Laboratory, Kirtland Air Force Base, NM.

${ }^{\ddagger}$ Professor, Department of Aerospace Engineering, The University of Michigan, Ann Arbor, MI.

$\S$ The research of the third author was supported in part by NSF award 1130160 and in part by the ASEE Summer Faculty Program.
} 
bank angle command $\sigma_{\text {des }}$. The reference flight path angle is then transformed to a reference angle-of-attack $\alpha_{\text {des }}$. Finally, the inner-loop system controls the vehicle to the commanded angle-of-attack and bank angle using the available longitudinal and lateral actuation.

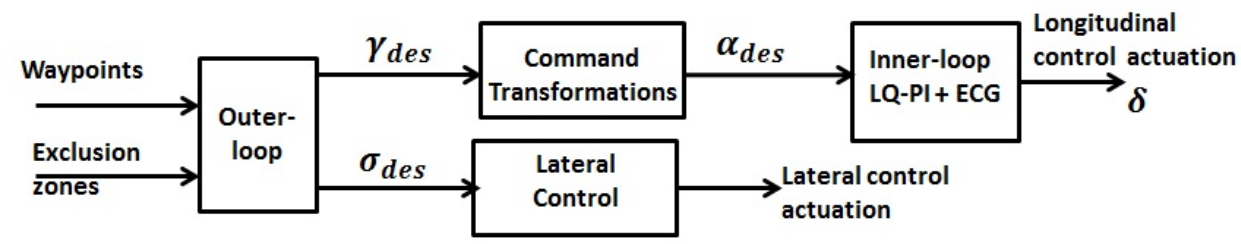

Figure 1. Framework including both the inner and outer-loop control schemes.

The outer-loop guidance layer implements a control approach based on a minimum-time Model Predictive Control formulation [8]. Such a controller provides the capability to guide the hypersonic vehicle through way points without violating the exclusion zone constraints that may or may not be known in advance. In addition, the MPC controller provides a robustness to the unmodelled dynamics [8], [9]. The innerloop control layer implements a control scheme for the longitudinal dynamics of a hypersonic glider with constraint enforcement capability [10]. In this loop, angle-of-attack command tracking is achieved using a Linear Quadratic Proportional plus Integral (LQ-PI) controller. An Extended Command Governor (ECG) is augmented to the nominal closed-loop system to handle the constraints.

In this paper, an integrated treatment of the outer and inner loop controllers are presented. Several extensions to the design of these controllers in [9] and [10] are also included. The outer-loop control design is discussed in Section II, where the MPC problem is formulated. In Section III, the inner-loop is considered. Its design includes a nominal LQ-PI controller augmented with the Extended Command Governor (ECG) to handle the pointwise-in-time state and control constraints. Section IV presents simulation results for the MPC outer-loop, the LQ-PI/ECG inner-loop, and a combination of both outer and inner loops. The paper is ended with concluding remarks.

\section{Outer-loop guidance using MPC}

For the outer-loop guidance layer, a simplified model for glider flight is used. Given this model, a minimum-time MPC scheme is developed to determine a reference flight path angle, $\gamma_{\text {des }}$, and a reference bank angle, $\sigma_{\text {des }}$, which the vehicle controls to while flying through a sequence of way points and avoiding exclusion zones. In some cases, the way points and exclusion zones may be known in advance. In other cases, they may only become known or may be modified in real-time during the flight.

\section{II.A. Model}

A simplified three dimensional model similar to the one in [11] for glider flight is used for MPC development. The starting point for this model, which depends on less parameters, are the three dimensional equations given in [12],

$$
\begin{aligned}
\frac{d x}{d t} & =V \cos (\beta), \\
\frac{d y}{d t} & =V \sin (\beta), \\
\frac{d h}{d t} & =V \gamma, \\
\frac{d V}{d t} & =-K_{D} V^{2} e^{-h / h_{0}}-g_{0} \gamma+\frac{V^{2} \gamma}{r_{0}+h}, \\
V \frac{d \gamma}{d t} & =K_{L} V^{2} e^{-h / h_{0}} \cos (\sigma)-g_{0}+\frac{V^{2}}{r_{0}+h}, \\
V \frac{d \beta}{d t} & =K_{L} V^{2} e^{-h / h_{0}} \sin (\sigma),
\end{aligned}
$$


where $x$ and $y$ are the coordinates of the glider, $h$ is the glider altitude, $\gamma$ is the glider flight path angle (assumed to be small), $h_{0}$ is the atmospheric scale height, $\sigma$ is the bank angle, and

$$
K_{L}=\frac{C_{L} A \rho_{0}}{2 m}, K_{D}=\frac{C_{D} A \rho_{0}}{2 m},
$$

with $\rho_{0}$ being atmospheric density at sea level, $C_{L}$ being the lift coefficient, $C_{D}$ being the drag coefficient, and $A$ being the glider cross-sectional area.

The equations in (1) can be simplified based on several assumptions. First, it is assumed that the desired flight path angle, $\gamma=\gamma_{d e s}$, is maintained by the inner-loop control of lift, resulting in $d \gamma / d t=0$. By the fifth equation of (1), it then follows that $K_{L}$ may be solved for, yielding

$$
K_{L}=\frac{g_{0}-V^{2} /\left(r_{0}+h\right)}{V^{2} \cos \sigma e^{-h / h_{0}}} .
$$

Under the assumption that (2) is maintained, the following set of equations are obtained,

$$
\begin{aligned}
\frac{d x}{d t} & =V \cos (\beta), \\
\frac{d y}{d t} & =V \sin (\beta), \\
\frac{d h}{d t} & =V \gamma_{d e s}, \\
\frac{d V}{d t} & =-K_{D} V^{2} e^{-h / h_{0}}-\gamma_{d e s}\left(g_{0}-\frac{V^{2}}{r_{0}+h}\right), \\
\frac{d \beta}{d t} & =\frac{c(V, h)}{V} \tan (\sigma),
\end{aligned}
$$

where

$$
c(V, h)=g_{0}-V^{2} /\left(r_{0}+h\right) .
$$

Next, $K_{L}$ and $K_{D}$ are linked by a single parameter based on the assumption that

$$
K_{D}=E \cdot K_{L} .
$$

From (2), (3), and (5),

$$
\begin{aligned}
\frac{d x}{d t} & =V \cos (\beta), \\
\frac{d y}{d t} & =V \sin (\beta), \\
\frac{d h}{d t} & =V \gamma_{d e s}, \\
\frac{d V}{d t} & =-c(V, h)\left(\frac{E}{\cos (\sigma)}+\gamma_{d e s}\right), \\
\frac{d \beta}{d t} & =\frac{c(V, h)}{V} \tan (\sigma) .
\end{aligned}
$$

These equations are convenient for the development of the inner-loop controller as they depend only on the single drag-to-lift parameter,

$$
E=\frac{K_{D}}{K_{L}}=\frac{C_{D}}{C_{L}},
$$

which may be time-varying during the flight. It should be noted that hypersonic gliders are designed as high lift-to-drag bodies, and as such, E will be relatively small. As a final step, the equations are nondimensionalized by employing a scaling transformation [11],

$$
x_{s}=\frac{x_{u}}{r_{0}}, \quad y_{s}=\frac{y_{u}}{r_{0}}, \quad h_{s}=\frac{h_{u}}{r_{0}}
$$




$$
\begin{aligned}
& \beta_{s}=\beta_{u}, \quad V_{s}=\frac{V_{u}}{\sqrt{g_{0} r_{0}}}, \\
& a_{s}=\frac{a_{u}}{g_{0}}, \quad t_{s}=\frac{t_{u}}{\sqrt{\frac{r_{0}}{g_{0}}}},
\end{aligned}
$$

where $(\cdot)_{s}$ stands for the transformed (scaled) variable and $(\cdot)_{u}$ stands for the untransformed variable in the original physical units. This leads to the following set of equations for the scaled variables (where we drop the subscript 's'),

$$
\begin{aligned}
\frac{d x}{d t} & =V \cos (\beta), \\
\frac{d y}{d t} & =V \sin (\beta), \\
\frac{d h}{d t} & =V \gamma_{d e s}, \\
\frac{d V}{d t} & =-\left(1-\frac{V^{2}}{1+h}\right)\left(\frac{E}{\cos (\sigma)}+\gamma_{d e s}\right), \\
\frac{d \beta}{d t} & =\left(\frac{1}{V}-\frac{V}{1+h}\right) \tan (\sigma) .
\end{aligned}
$$

Since equations (7) hold under the assumption (2), a maximum lift constraint is enforced by the MPC controller,

$$
K_{L}=\frac{1-V^{2} /(1+h)}{V^{2} r_{0} \cos (\sigma) e^{-r_{0} h / h_{0}}} \leq K_{L, \max } .
$$

In addition, a nondimensional stagnation heating rate constraint from [11] is considered in the following form,

$$
\frac{k_{d i m} e^{-r_{0} h / h_{0}} V^{3}}{\sqrt{r_{\text {nose }}} \dot{q}_{s, \text { max }}}-1 \leq 0,
$$

where $k_{d i m}$ is a heating rate constant, $r_{n o s e}$ is the vehicle nose radius, and $\dot{q}_{s, \text { max }}$ is the dimensional maximum heat flux.

\section{II.B. MPC Design for Three Dimensional Model to Control Bank Angle}

In this section, the MPC design for the bank angle control is described, assuming that $\gamma_{\text {des }}$ is prescribed by a non-MPC controller as a constant value. This design provides a conceptually and computationally simpler starting point for MPC development as has advantages from a practical implementation standpoint. In later sections of this paper, the design will be extended to prescribing both desired bank angle, $\sigma_{d e s}$, and desired flight path angle, $\gamma_{\text {des }}$.

In reference to the three-dimensional model (7), the way points are defined by their positions along the $x$ and $y$ axes. The $x$ and $y$ coordinates of the way point the glider is flying to at current time $t$ are denoted, respectively, by $x_{f}(t)$ and $y_{f}(t)$. For the hypersonic vehicle outer-loop, a minimum-time formulation of Model Predictive Control with two phases is used. The first is known as the 'fly to' the way point phase while the second is called the 'fly through' the way point phase. When the distance between the glider and the way point is above a specified threshold, the 'fly to' mode is active. Once this distance falls below the specified threshold, the 'fly through' mode is activated.

In both phases, the cost function

$$
J=T_{f}+\rho\left(\left(x\left(t+T_{f} \mid t\right)-x_{f}\right)^{2}+\left(y\left(t+T_{f}\right)-y_{f}\right)^{2}\right)+\mu\left(\phi\left(t+T_{f} / N \mid t\right), \quad \rho, \mu \geq 0 .\right.
$$

is minimized subject to the constraints

$$
\begin{gathered}
\tau=0, \frac{T_{f}}{N}, \frac{2 T_{f}}{N}, \ldots T_{f}, \\
\tan (\sigma)=\tan (60 \pi / 180) u, \quad-1 \leq u(t+\tau \mid t) \leq 1,
\end{gathered}
$$




$$
\begin{gathered}
\left|u\left(t+\tau_{i} \mid t\right)-u\left(t+\tau_{i+1} \mid t\right)\right| \leq 0.2, \quad i=1, \ldots, N-1, \\
x(t+\tau \mid t) \notin E_{i}, 0 \leq \tau \leq T_{f}, \quad i=1, \ldots, n_{E},
\end{gathered}
$$

where $t$ denotes the current time, $t+T_{f}$ is the final time, $T_{f}$ is the prediction horizon (not fixed), $\phi$ is the absolute difference between the angle to the way point and the heading angle $\beta, \rho$ and $\mu$ are constant weights, $\xi(\tau \mid t)$ denotes the predicted value of a variable $\xi$ at time $\tau$ while the prediction is made at time $t$, $N$ is the number of steps in the time horizon, and $E_{i}$ denotes the $i$ th exclusion zone, $i=1, \ldots, n_{E}$. The exclusion zones are defined as ellipsoidal sets,

$$
E_{i}=\left\{(x, y): \frac{1}{2}\left[x-x_{E, i} \quad y-y_{E, i}\right] P_{i}\left[x-x_{E, i} \quad y-y_{E, i}\right]^{\mathrm{T}} \leq 1\right\},
$$

where $P_{i}=P_{i}^{\mathrm{T}}>0$. In [11], the exclusion zones are disks and $P_{i}=\alpha I_{2}, \alpha>0$, where $I_{2}$ is the $2 \times 2$ Identity matrix. Note that the constraints (14)-(15) are non-convex.

The differences between the 'fly to' phase and 'fly through' phases lie within the slack variable $\rho$. In the 'fly to' phase, $\rho$ is used to softly enforce the constraint for the predicted trajectory to terminate at the way point. Once the 'fly through' phase is activated at a time $t_{a}$, the optimization problem (10)-(14) is solved once, with the terminal way point constraint,

$$
x\left(t+T_{f} \mid t\right)=x_{f}(t), \quad y\left(t+T_{f} \mid t\right)=y_{f}(t),
$$

imposed and the optimal trajectory $u^{*}\left(t_{a}+\tau \mid t_{a}\right)$ executed open-loop. As the glider is close to the way point at a point of activation of the 'fly through' phase, following the open-loop trajectory does not significantly degrade the robustness. Once the open-loop trajectory is completed, the controller switches to the 'fly to' mode and flies to the next way point. In order to realize this approach, ellipsoidal switching or 'fly through zones' are introduced around the way points. The use of a two phase system is a simpler strategy than used in previous works [9]. Future work will be done to determine the impact on performance and robustness versus the strategies considered in [9].

To treat the MPC problem (10)-(14) numerically, the time is scaled as $s=\tau / T_{f}$, so that $0 \leq s \leq 1$, and the model, represented compactly as $\dot{x}=d x / d \tau=f(x, u)$, is re-written as

$$
x^{\prime}=\frac{d x}{d s}=\frac{d x}{d \tau} \frac{d \tau}{d s}=T_{f} f(x, u)
$$

For the model in (17), the optimal control problem has to be solved over a fixed time horizon of 1 . Therefore, $T_{f}$ becomes a parameter that needs to be optimized along with the control, $u(\cdot)$. The scaled time interval of control, $[0,1]$, is partitioned into $N$ equal intervals of length $\Delta s=\frac{1}{N}$. The control values $u\left(s_{i}\right)$, at $s_{i}=\Delta s_{i}$, $i=0, \cdots, N-1$, are optimized along with $T_{f}$, under the Zero Order Hold assumption that $u(s)=u\left(s_{i}\right)$ for $s_{i} \leq s<s_{i+1}$.

\section{Inner-loop control using LQ-PI/ECG}

The inner-loop controller of the hypersonic glider realizes the commanded bank angle and flight path angle by commanding an angle-of-attack. In this Section, the constrained inner-loop control of the angle-ofattack is exemplified. For this, a nominal LQ controller for pitch is used to track the reference angle-of-attack while an Extended Command Governor is utilized to modify the command to ensure that the constraints are not violated, especially the elastic defection constraints, which are of particular interest. The design of ECG is based on [13]. As compared to reference governor designs such as the one proposed in [6], ECG can provide increased constrained domains of attraction, faster responses, and increased tolerances to uncertainties, even without explicit disturbance and uncertainty in the model. From the standpoint of control design, ECG is attractive, as it augments but does not replace the nominal controller. The model used for the design on the LQ controller is from [14]. 


\section{III.A. Longitudinal equations of motion for a flexible hypersonic glider}

The longitudinal equations of motion, based on [14], are,

$$
\begin{aligned}
\dot{V}_{t} & =\frac{T \cos \alpha-D}{m-g \sin \gamma}, \\
\dot{\alpha} & =-\frac{T \sin \alpha+L}{m V_{t}}+\left(\frac{g}{V_{t}}-\frac{V_{t}}{r}\right) \cos \gamma+Q, \\
\dot{Q} & =\frac{\mathcal{M}}{I_{y y}} \\
\dot{\Theta} & =Q \\
\dot{h} & =V_{t} \sin \gamma, \\
\ddot{\eta}_{i} & =-2 \xi \omega_{i} \dot{\eta}_{i}-\omega_{i}^{2} \eta_{i}+N_{i}, i=1,2, \ldots, n,
\end{aligned}
$$

where $V_{t}$ is the true airspeed, $T$ is the thrust, $D$ is the drag, $L$ is the lift, $\alpha$ is the angle-of-attack, $\gamma$ is the flight path angle, $\mathcal{M}$ is the pitching moment, $Q$ is the pitch rate, $h$ is the altitude, and $r$ is the radius of the vehicle. The flexible dynamics of the vehicle are modeled in (23), where $\eta_{i}$ is the ith modal coordinate of the flexible dynamics, $\xi$ is the damping ratio, $\omega_{i}$ is the natural frequency, and $N_{i}$ is the ith generalized force. The modal method and its derivation is more completely described in [14].

\section{III.B. Constraints}

Given the model in the previous section, constraints were added to reflect requirements relevant to maintaining vehicle flight. These constraints include the angle-of-attack, pitch rate, elevator deflection, and elevator deflection rate, represented by

$$
\begin{gathered}
\alpha_{\min } \leq \alpha(t) \leq \alpha_{\max }, \\
Q_{\min } \leq Q(t) \leq Q_{\max }, \\
\delta_{e_{\min }} \leq \delta_{e}(t) \leq \delta_{e_{\max }}, \\
\Delta \delta_{e_{\min }} \leq \Delta \delta_{e}(t) \leq \Delta \delta_{e_{\max }} .
\end{gathered}
$$

The angle-of-attack is constrained so that the lift forces acting on vehicle do not allow it to transition to uncontrollable flight, which may occur at large angles-of-attack. The elevator deflection is constrained due to its limited range of travel; in practice, tighter constraints to maintain the glider within a controllable flight range may be imposed. The rate of elevator deflection is constrained to reflect the actuator's physical limits and also to ensure safe control by preventing maneuvers that can lead to unstable flight.

Due to the glider's high speeds in the hypersonic flight regime, structural dynamics, including aeroelastic effects, play a significant role. Constraints must be imposed in order to ensure acceptable elastic deflections. Specifically, at specified distances back from the nose of the vehicle, the contributions of the three modal frequencies are constrained to be less than a specific value

$$
E D_{\min } \leq \sum_{i=1}^{3} \Phi_{i}\left(x_{l o c, i}\right) \eta_{i}(t) \leq E D_{\max }
$$

where $E D$ is the total elastic deflection of the vehicle and $\Phi_{i}$ is the ith mode shape at the specified location on the vehicle, $x_{l o c, i}$. This constraint enables the designed controller to keep the vehicle's structure safe during the gliding portion of flight. The selected locations for this paper are at 1/10,3/10,5/10, 7/10 and $9 / 10$ of vehicle length from the nose.

\section{III.C. LQ-PI Controller with Extended Command Governor}

Given the model proposed above, a nominal LQ-PI controller is augmented with an Extended Command Governor such that the previously mentioned constraints can be met. The equations of motion given are linearized about a trim point assuming zero thrust for the vehicle. This yields an 11 dimensional system with the first five states given above (velocity $V$, angle-of-attack $\alpha$, pitch rate $Q$, altitude $h$ and pitch altitude $\theta)$ and the last six are the modal coordinates and their time derivatives $\left(\eta_{1}, \dot{\eta}_{1}, \eta_{2}, \dot{\eta}_{2}, \eta_{3}, \dot{\eta}_{3}\right)$. The control input for the system is the elevator deflection, $\delta_{e}$. 


\section{III.C.1. LQ-PI controller}

The LQ-PI controller is designed using the short period subsystem extracted from the continuous-time linearized system model. This subsystem includes the angle-of-attack, pitch rate, the modal coordinates, and their derivatives in the state vector $x_{s s} \in \mathbf{R}^{8}$, and has the following representation

$$
\dot{x}_{s s}=\boldsymbol{A}_{s s} x_{s s}+\boldsymbol{B}_{s s} u,
$$

where $u$ denotes the elevator position and $x_{s s}$ is the state deviation from the trim conditions. The controller, designed for set-point tracking of angle-of-attack commands, has a tracking error

$$
e=\alpha-\alpha_{\text {des }} .
$$

The control law for $u$ has the following form

$$
u=-K_{1} \int_{0}^{t} e(\tau) d \tau-K_{2} x_{s s},
$$

where $K_{1}$ is the integral gain and $K_{2}$ is the proportional gain.

The augmented system used to design the LQ-PI controller has the form

$$
\dot{x}_{a u g}=\boldsymbol{A}_{\text {aug }} x_{a u g}+\boldsymbol{B}_{\text {aug }} \dot{u},
$$

where

$$
x_{a u g}=\left[\begin{array}{c}
e \\
\dot{x}
\end{array}\right], \boldsymbol{A}_{a u g}=\left[\begin{array}{cc}
0 & \boldsymbol{C}_{\boldsymbol{\alpha}} \\
0 & \\
\vdots & \boldsymbol{A}_{\boldsymbol{s s}} \\
0 &
\end{array}\right], \boldsymbol{B}_{\text {aug }}=\left[\begin{array}{c}
0 \\
\boldsymbol{B}_{\boldsymbol{s s}}
\end{array}\right],
$$

and $\alpha=\boldsymbol{C}_{\boldsymbol{\alpha}} x_{s s}$ is the angle-of-attack output. It is assumed that when deriving (33), the set-point is constant, i.e., $\dot{\alpha}_{\text {des }}=0$.

The LQR design was based on the matrix $Q$ set to

$$
Q=\left[\begin{array}{cccc}
1 & 0 & \cdots & 0 \\
0 & & & \\
\vdots & & 0 & \\
0 & & &
\end{array}\right]
$$

where $\mathbf{0}$ is an $8 \times 8$ zero matrix and $R$ is set to 0.00005 . The controller tracks asymptotically the angle-ofattack command with fast response and small overshoot. To treat the case when the elastic states are not measured or estimated, the second LQ-PI controller was designed based on the second order subsystem (32) with states being the angle-of-attack and pitch rate. In this case, the LQ-PI controller uses feedback on the integral of the angle-of-attack, angle-of-attack tracking error, and pitch rate.

\section{III.C.2. Extended Command Governor Design}

An ECG is implemented to enforce state and control constraints by modifying the set-point commands to the closed-loop system, as shown in Fig. 2. In this system, $\hat{x}$ is the state estimate, $y$ is the system output constrained by the set inclusion conditions $y(t) \in Y$ for all $t, w$ is the disturbance/uncertainty, $v$ is the ECG output, and $r$ is the reference command. In this paper, $w(t)=0$ as the characterization and treatment of uncertainty is left to future publications.

The ECG uses the set, $\check{O}_{\infty}$, of states and parameterized inputs such that the constraints are satisfied for all time. The ECG output is defined by

$$
v(t)=\rho(t)+\bar{C} \bar{x}(t)
$$

where $\rho(t)$ and $\bar{x}(t)$ are solutions to the following optimization problem

$$
\begin{array}{ll}
\operatorname{minimize} & \|\rho(t)-r(t)\|_{Q}^{2}+\|\bar{x}(t)\|_{S}^{2}, \\
\text { subject to } & (\rho(t), \bar{x}(t), x(t)) \in \check{O}_{\infty} .
\end{array}
$$




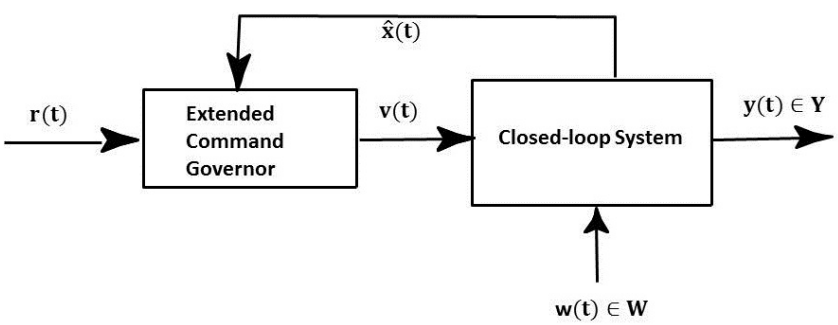

Figure 2. Extended command governor applied to a closed-loop system.

The $\bar{x} \in \mathbf{R}^{\overline{\mathbf{n}}}, \bar{n} \geq 0$, and $\rho$ are states of a stable auxiliary dynamic system which evolve over the semi-infinite prediction horizon according to

$$
\begin{gathered}
\bar{x}(t+k+1)=\bar{A} \bar{x}(t+k), k \geq 0 \\
\rho(t+k)=\rho(t) .
\end{gathered}
$$

Compared to reference governors and conventional command governors (where $\bar{n}=0$ ), the ECG yields a larger domain of recoverable initial states and faster response.

The set $\check{O}_{\infty}$ is a finitely determined inner approximation to the set of all $(\rho(t), \bar{x}(t), x(t))$ that do not induce subsequent constraint violation when the input sequence $v(t+k)$ is determined by the fictitious dynamics per (35) and (37). In the case when $Y$ is polyhedral, computational procedures exist that lead to polyhedral $\breve{O}_{\infty}$ [15]. Without the fictitious states, i.e., when $\bar{n}=0$, the ECG becomes the simple command governor [16]. The optimization problem can be solved online using conventional quadratic programming techniques. The iterative procedures can be avoided by using explicit multi-parametric quadratic programming [17], leading to $\rho$ and $\bar{x}$ being given as a piecewise-affine function of the state $x(t)$ and reference $r$.

Various choices of $\bar{A}$ and $\bar{C}$ can be made. The shift sequences used in [16] are generated by the fictitious dynamics with,

$$
\begin{aligned}
\bar{A} & =\left[\begin{array}{ccccc}
0 & I_{m} & 0 & 0 & \ldots \\
0 & 0 & I_{m} & 0 & \ldots \\
0 & 0 & 0 & I_{m} & \ldots \\
0 & 0 & 0 & 0 & \ldots \\
\vdots & \vdots & \vdots & \vdots & \ddots
\end{array}\right] \\
\bar{C} & =\left[\begin{array}{ccccc}
I_{m} & 0 & 0 & 0 & \ldots
\end{array}\right],
\end{aligned}
$$

where $I_{m}$ is an $m \times m$ identity matrix. Another approach uses Laguerre sequences [18]. These sequences possess orthogonality properties and are generated by the fictitious dynamics with

$$
\begin{gathered}
\bar{A}=\left[\begin{array}{ccccc}
\epsilon I_{m} & \zeta I_{m} & -\epsilon \zeta I_{m} & \epsilon^{2} \zeta I_{m} & \cdots \\
0 & \epsilon I_{m} & \zeta I_{m} & -\epsilon \zeta I_{m} & \cdots \\
0 & 0 & \epsilon I_{m} & \zeta I_{m} & \cdots \\
0 & 0 & 0 & \epsilon I_{m} & \cdots \\
\vdots & \vdots & \vdots & \vdots & \ddots
\end{array}\right] \\
\bar{C}=\sqrt{\zeta}\left[\begin{array}{llllll}
I_{m} & -\epsilon I_{m} & \epsilon^{2} I_{m} & -\epsilon^{3} I_{m} & \cdots & (-\epsilon)^{N-1} I_{m}
\end{array}\right],
\end{gathered}
$$

where $\zeta=1-\epsilon^{2}$ and $0 \leq \epsilon \leq 1$ is a selectable parameter that corresponds to the time-constant of the fictitious dynamics. Note that with the choice of $\epsilon=0,(39)$ coincides with the shift register considered in [16]. The implementation of the ECG is feasible using partial state information and reduced order models (e.g., without the elastic dynamics) following the approach in [19]. 
For this work, the constraints are on angle-of-attack, pitch rate, elevator deflection, elevator deflection rate, and elastic deflection. The discrete-time model of the closed-loop system necessary to implement ECG is based on the LQ-PI controller (one of two designs depending on whether elastic modes are used for feedback), the short period subsystem (with elastic modes), and a sampling period of $0.01 \mathrm{sec}$. This model has the following form

$$
\begin{aligned}
& x(t+1)=A x(t)+B v(t), \\
& y(t)=C x(t)+D v(t) \in Y .
\end{aligned}
$$

\section{Simulations}

The following section presents multiple simulation cases. In Subsection IV.A, the outer-loop results for MPC control of bank angle is presented. Subsection IV.B presents results of the LQ-PI/ECG inner-loop control of angle-of-attack. The outer-loop and inner-loop are then combined, with simulations shown in Subsection IV.C. Finally, the MPC controller is modified to control both bank angle and flight path angle in Subsection IV.D. Including the flight path angle to the MPC controller adds computational complexity, but enables 3D travel to way points and 3D exclusion zone avoidance.

\section{IV.A. MPC Simulation Results}

The simulation results for $\gamma_{d e s}=-\frac{\pi}{180}(-1$ degree $)$ are shown in Figs. 3-6. The discretization of this control trajectory is based on $N=8$ and the cost (10) uses $\rho=1$ and $\mu=1$. The trajectory of the glider in the $x-y$ plane is shown in Fig. 3, with exclusion zones shown as large red disks, 'fly-through' regions identified as medium size empty circles, and small circles illustrating the 'fly through' portion of the trajectory. The simulation scenario assumes that the glider initially flies straight along $y$-axis while exclusion zones, centered at $(-0.2,-0.05),(-0.7,-0.45)$, and $(0.3,0.45)$, appear on the glider path along to way point 1 at $(-0.3,0.3)$. Due to the constraint in (15), the glider is banked to steer away from the exclusion zone on its path. Based on the assumed parameters of the problem in the numerical simulation, the glider's velocity and altitude rapidly decrease. Hence, it is assumed that once $h$ decreases to 0.01 , the flight path angle is changed to $\gamma_{d e s}=0$, and once $V$ decreases to 0.1 , powered flight is initiated with $\dot{V}=0$ and $\gamma_{d e s}=0$. On hypersonic vehicles, this powered flight is provided by boosters or scramjet propulsion. The change from a steady decreasing altitude to a flat constant altitude reflects the change from a $\gamma_{\text {des }}$ of $-\frac{\pi}{180}$ to that of 0 . The powered phase of the flight, near the end of the flight, is identified in Fig. 3 by a superimposed yellow line. It corresponds to phases where the trajectories of $V$ and $h$ become flat.

In addition, the lift and stagnation heating rates are shown in Fig. 6 . The lift parameter $K_{L}$ has a max value at $K_{L, \max }=2.94 \times 10^{-4}$ per meter while the maximum nondimenisonal heating rate is 0 (see (9)). The glider's $K_{L}$ in this simulation reaches its maximum when altitude and velocity are at their minimum values, but it is shown that this value does not exceed $K_{L, \max }$. The stagnation heating rate rapidly increases as the glider descends, and reaches its maximum when the altitude is at its lower limit. After the altitude limit is met, the heating rate decreases and becomes constant.

\section{IV.B. ECG Simulation results}

To demonstrate the effect of the ECG, an example scenario is constructed with the limits as follows: $-\alpha_{\min }=$ $\alpha_{\max }=0.3 \mathrm{rad}$ for the angle of attack; $-Q_{\min }=Q_{\max }=0.2 \mathrm{rad} / \mathrm{sec}$ for the pitch rate,$-\delta_{e, \min }=\delta_{e, \max }=$ $0.349 \mathrm{rad}$ for the elevator deflection $(20 \mathrm{deg}),-\Delta \delta_{e, \min }=\Delta \delta_{e, \max }=0.349 \mathrm{rad} / \mathrm{sec}$ for the elevator deflection rate, and $-E D_{\min }=E D_{\max }=1.0$ for the elastic deflections. Fig. 7 shows the effect of implementing the ECG to handle constraints on angle-of-attack. The elevator deflection of the system for the system with and without the ECG is shown in Fig. 8, and the elastic deflection is shown in Fig. 9.

\section{IV.C. MPC and ECG Control of Bank Angle and Angle-of-Attack}

The outer-loop MPC and inner-loop LQ-PI/ECG are combined by transforming the outer-loop flight path angle command to a reference angle-of-attack command for the inner-loop. This transformation is computed by utilizing pitch and flight path angle tracking to obtain a commanded angle-of-attack.

The simulation in this Subsection is similar to the flight simulation in Subsection IV.A. The glider must fly to a single way point centered at $(-0.3,0.3)$ while avoiding several large exclusion zones centered at 


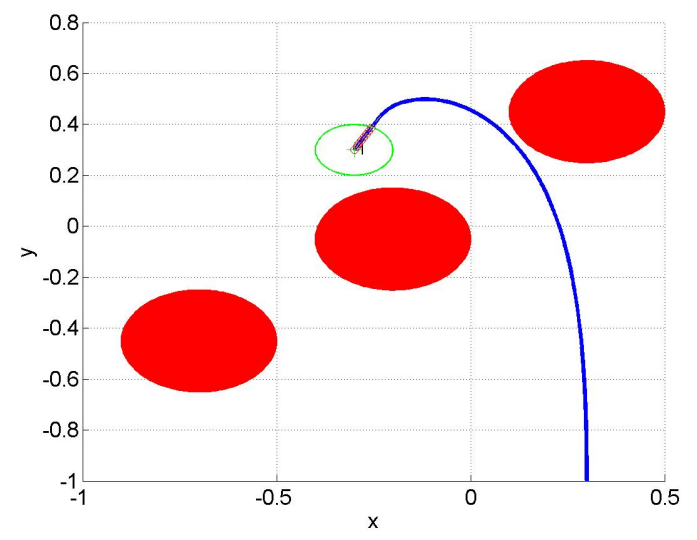

(a)
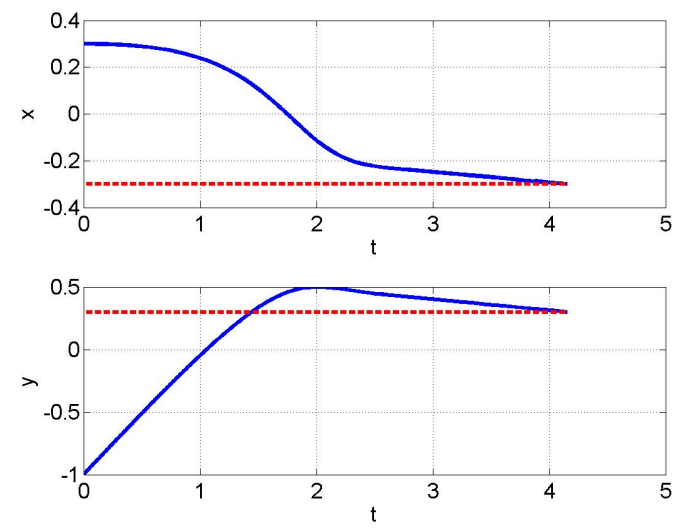

(b)

Figure 3. Left: Glider trajectory in the $x-y$ plane. Right: The time histories of $x$ and $y$. Dashed lines show the target positions as implied by the specified way points.

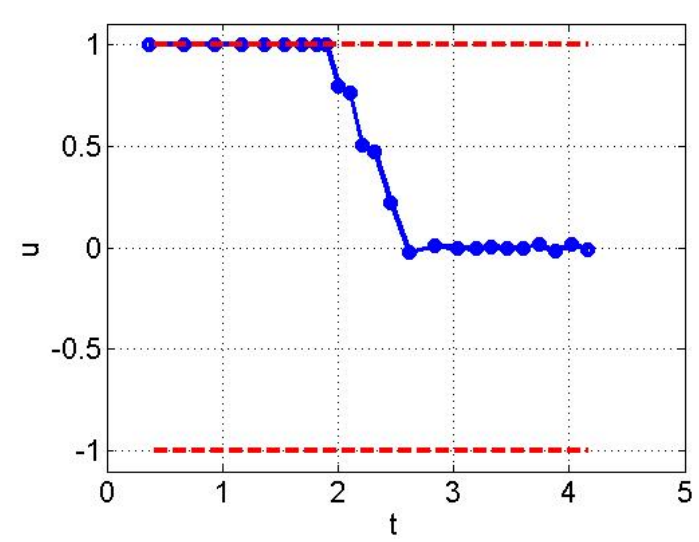

(a)

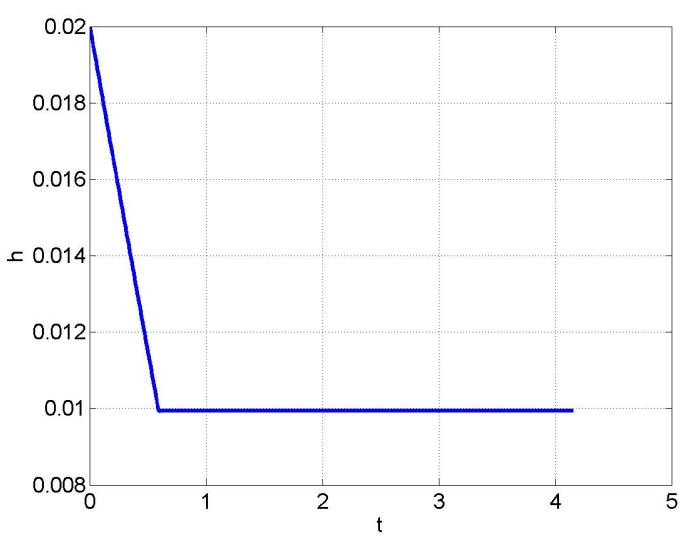

(b)

Figure 4. Left: Time history of normalized bank angle control signal. Note: bank angle sampled values are shown at the end of each control interval. Right: Time history of glider altitude.

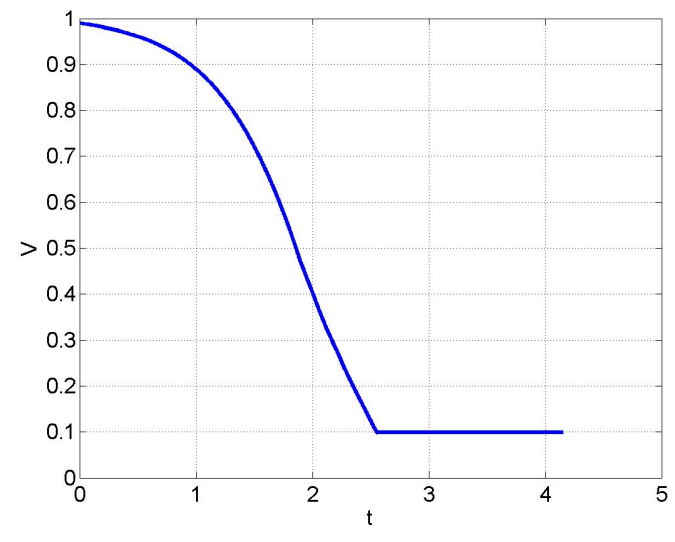

(a)

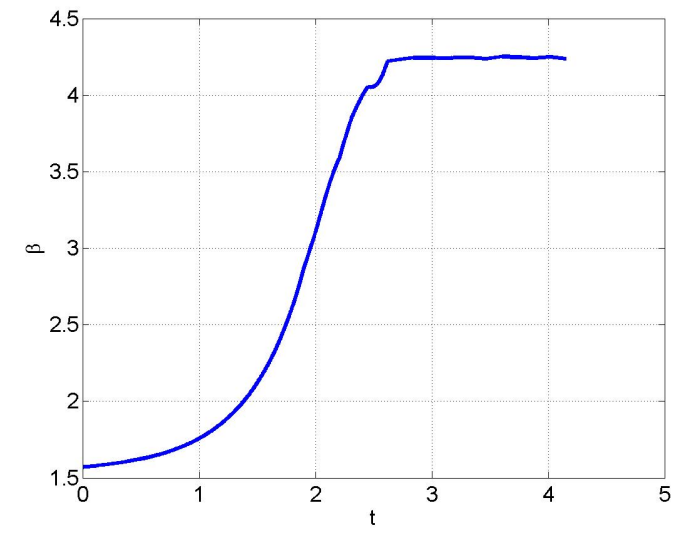

(b)

Figure 5. Left: Time history of glider velocity. Right: Time history of glider heading angle. 


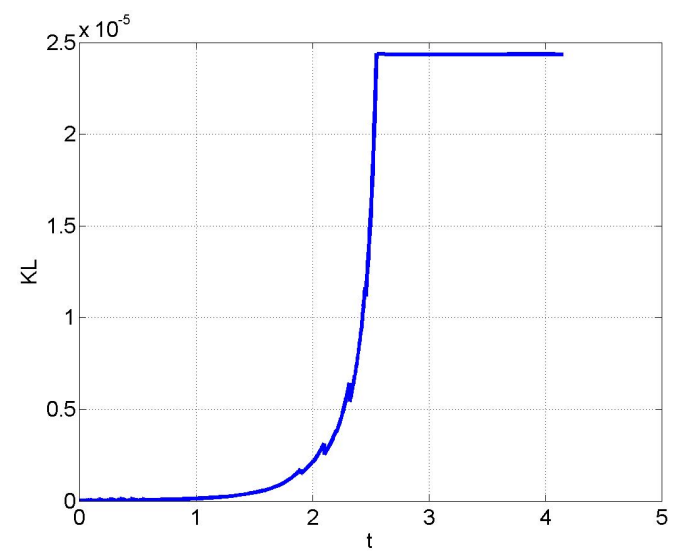

(a)

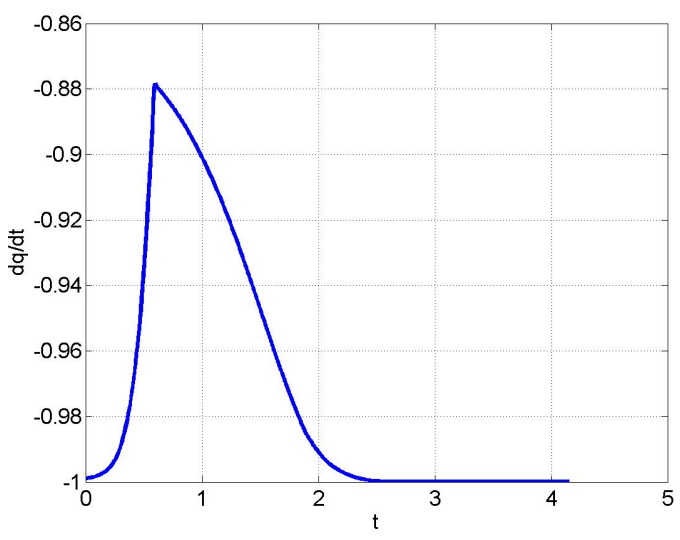

(b)

Figure 6. Left: Time history of glider lift coefficient $K_{L}$. Right: Time history of undimensional glider heating rate.

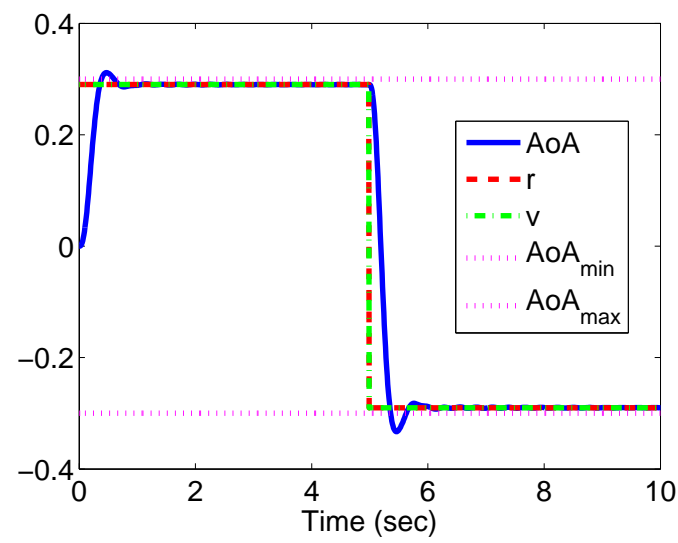

(a)

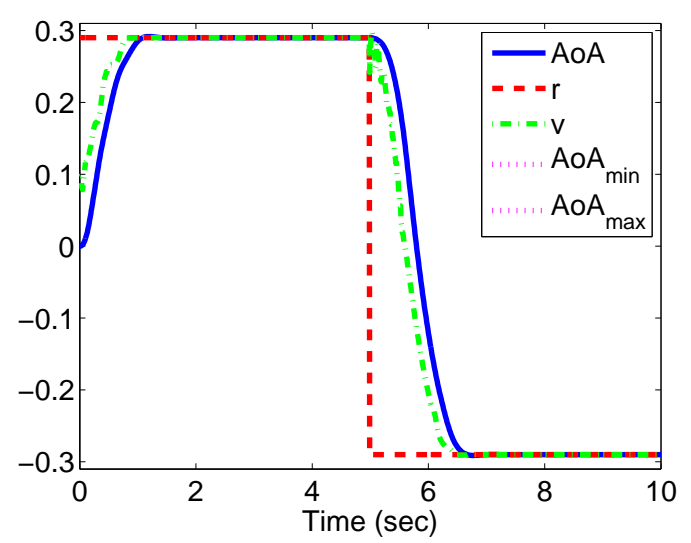

(b)

Figure 7. Left: The response with and without the ECG for angle-of-attack. Right: The response with the ECG formulated for $\bar{n}=5, \epsilon=0$.

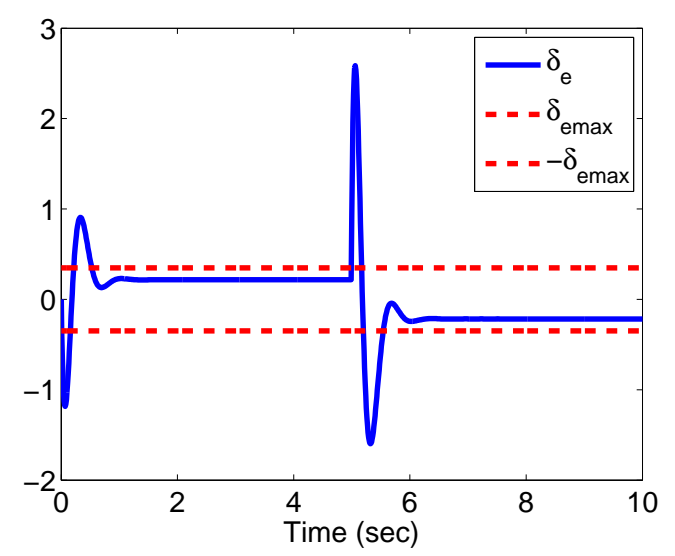

(a)

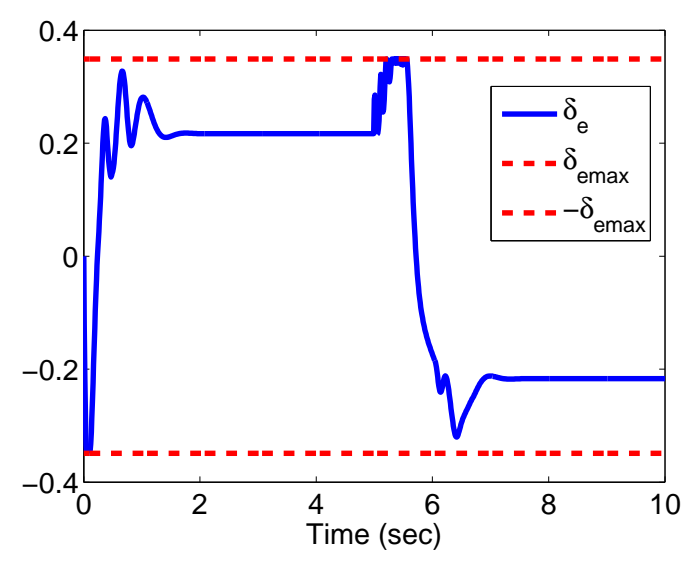

(b)

Figure 8. Left: The response with and without the ECG for elevator deflection. Right: The response with the ECG formulated for $\bar{n}=5, \epsilon=0$. 


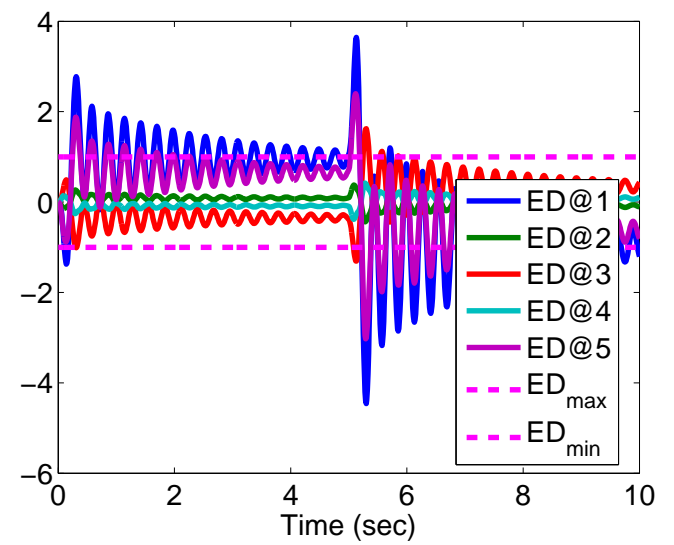

(a)

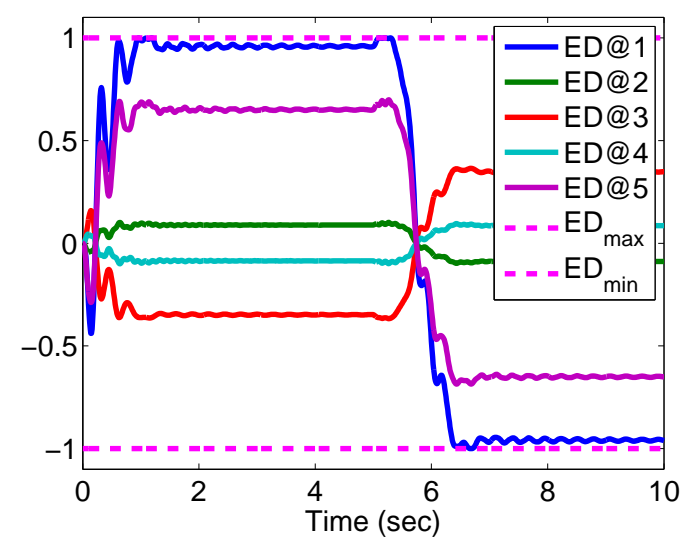

(b)

Figure 9. Left: The response with and without the ECG for elastic deflection. Right: The response with the ECG formulated for $\bar{n}=5, \epsilon=0$.

$(-0.2,-0.05),(-0.7,-0.45)$, and $(0.3,0.45)$. The difference in the MPC outer-loop is that $\gamma_{\text {des }}=-\frac{2 \pi}{180}(-2$ degrees) as long as $h \geq 0.007$ and $\gamma_{\text {des }}=0$ afterwards in order to excite angle-of-attack control. The ECG has the same constraints as used previously.

From the results presented in Figs. 10-14, it can be seen that all control and structural constraints have been satisfied in order to ensure safe hypersonic flight. The lift and stagnation heating constraints do not reach their limits, and the patterns of heating and lift are the same as the previous simulation with just MPC. The excitation in the longitudinal dynamics occurs during the initial stage of flight, when the ECG is commanding various angles-of-attack in order to achieve a desired flight path angle. Once the glider reaches its desired flight path angle, the longitudinal dynamics have constant values. Due to the small angle-ofattack, the values for elevator deflection, elevator deflection rate, and elastic deflection are all small, well within the predefined limits.

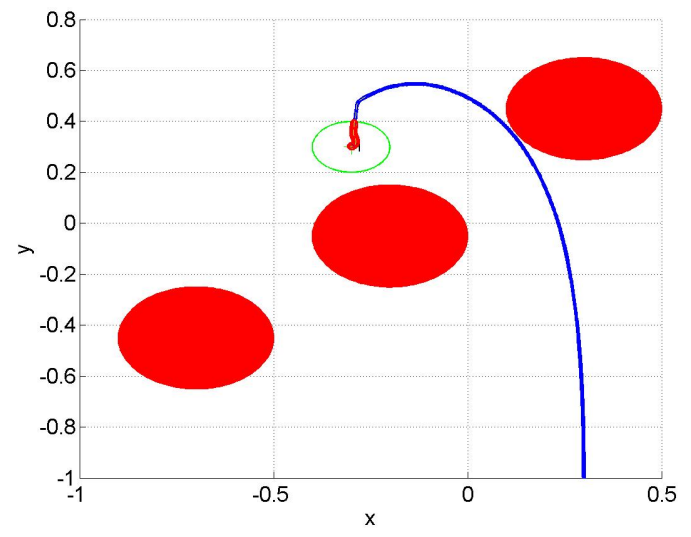

(a)

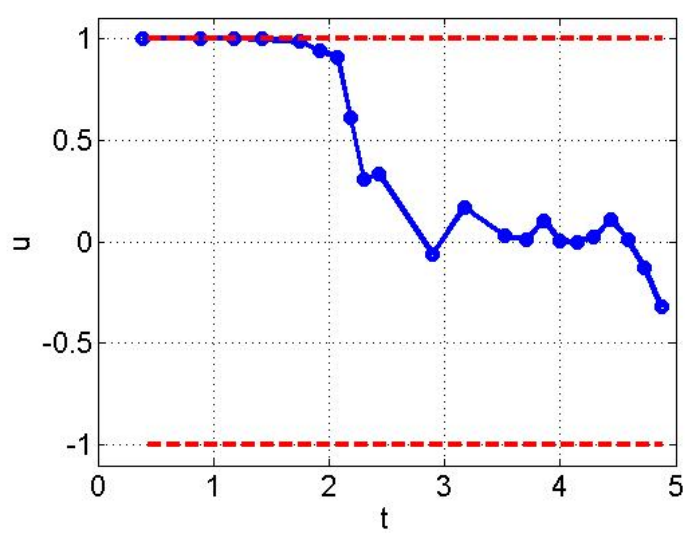

(b)

Figure 10. Left: MPC and ECG glider trajectory in the $x-y$ plane. Right: Time history of normalized bank angle control signal using MPC and ECG. Note: bank angle sampled values are shown at the end of each control interval.

\section{IV.D. MPC and ECG Control of Bank Angle and Angle-of-Attack}

In earlier sections of this paper, the flight path angle was kept constant while the bank angle was optimized. In order to add flight path angle control to the MPC framework, an altitude factor had to be added to the cost function, the fly through zones, and the exclusion zones. Control of both bank angle and flight angle is more computationally exhaustive then just bank angle control, but it allows for 3D way point travel and 


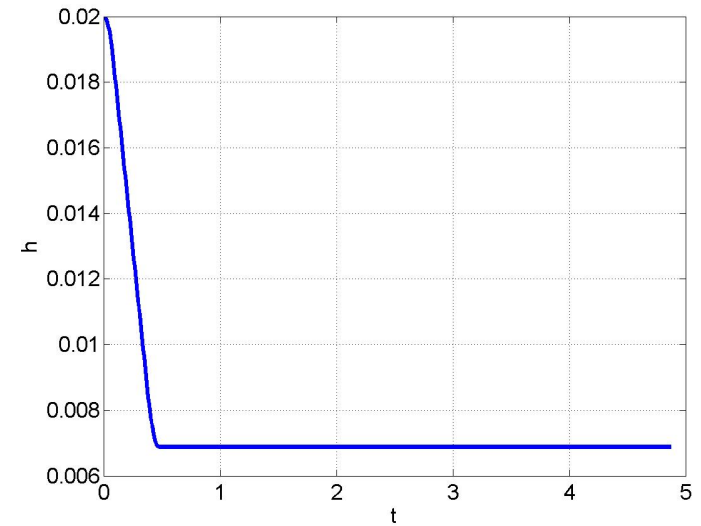

(a)

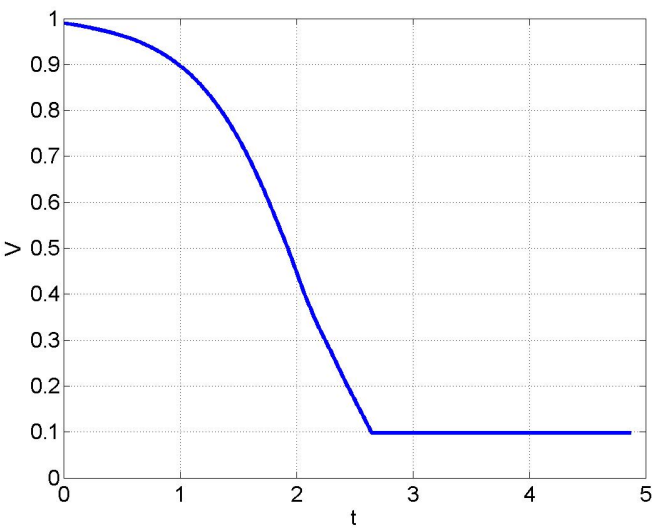

(b)

Figure 11. Left: Time history of glider altitude with MPC and ECG. Right: Time history of glider velocity with MPC and ECG.

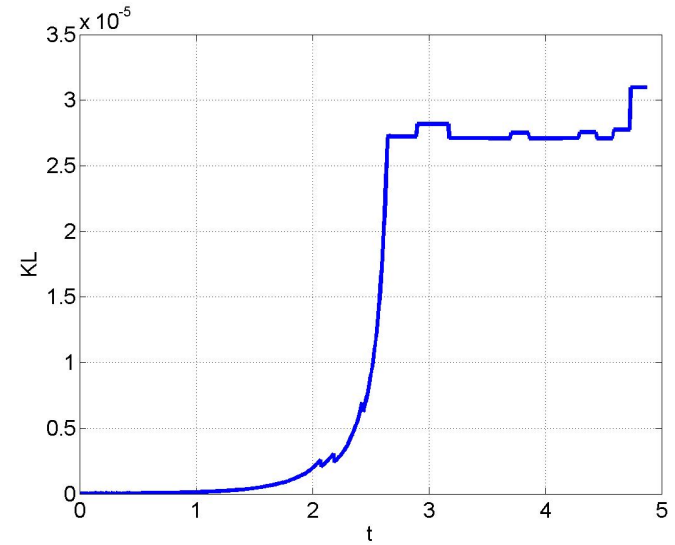

(a)

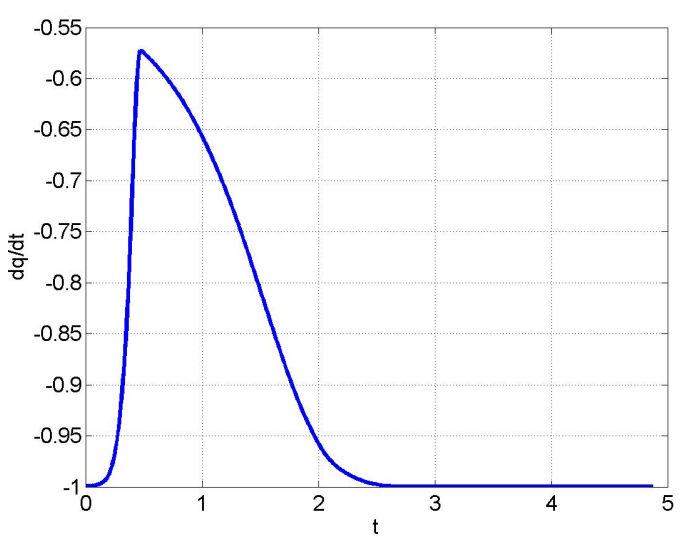

(b)

Figure 12. Left: Time history of glider lift coefficient $K_{L}$ using MPC and ECG. Right: Time history of undimensional glider heating rate using MPC and ECG.

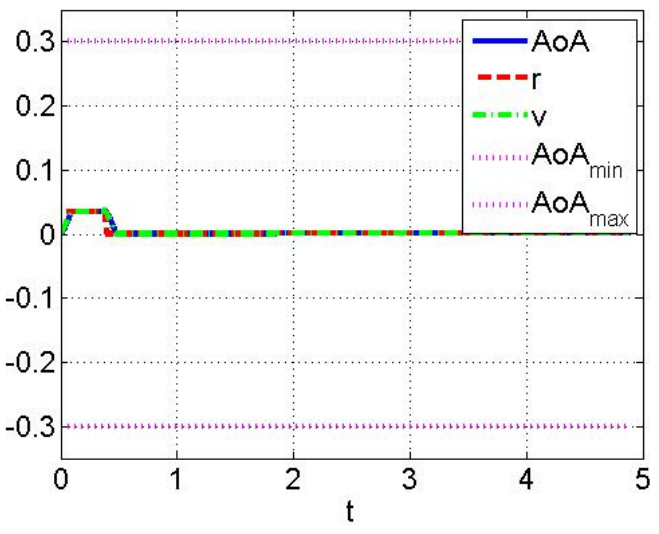

(a)

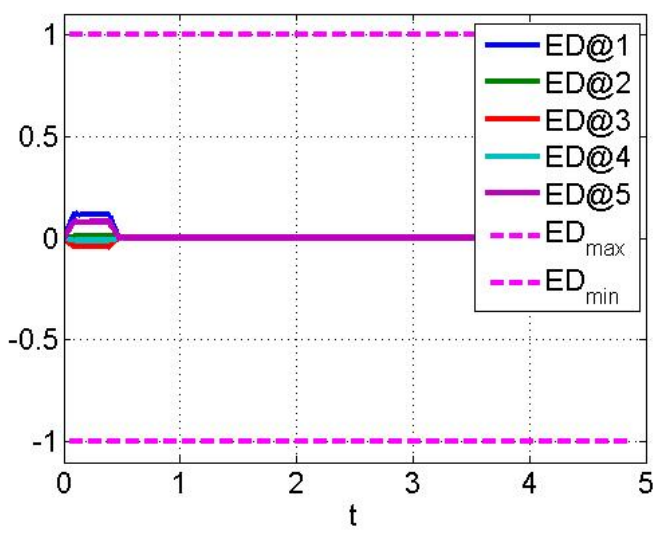

(b)

Figure 13. Left: The response of angle-of-attack (solid) with constraints. Right: The response of elastic deflection and constraints. 


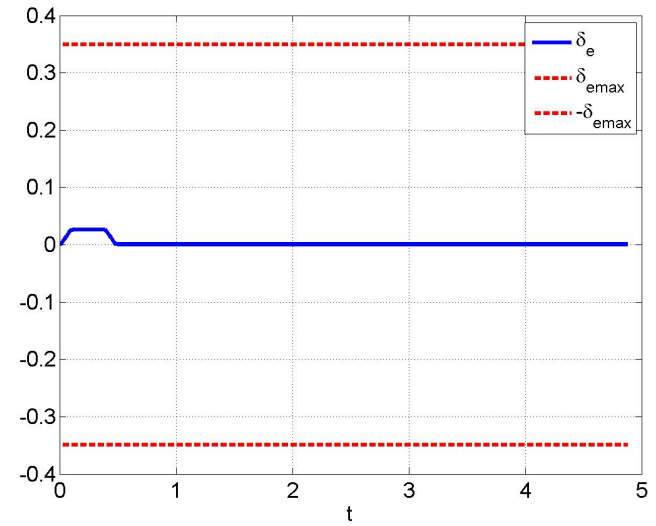

(a)

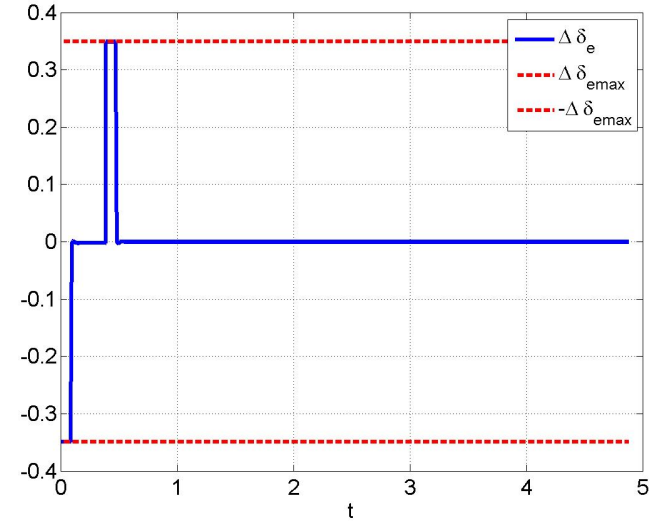

(b)

Figure 14. Left: The response of elevator deflection with constraints. Right: The response of elevator deflection rate with constraints.

exclusion zone avoidance. The cost function with altitude included is

$$
J=T_{f}+\rho\left(\left(x\left(t+T_{f} \mid t\right)-x_{f}\right)^{2}+\left(y\left(t+T_{f}\right)-y_{f}\right)^{2}+\left(h\left(t+T_{f} \mid t\right)-h_{f}\right)^{2}\right)+\mu\left(\phi\left(t+T_{f} / N \mid t\right), \quad \rho, \mu \geq 0 .\right.
$$

The switching zones between the fly to and the fly through modes around the way points are defined as ellipsoids given by

$$
W P_{i}=\left\{(x, y, h):\left[\begin{array}{lll}
x-x_{W P, i} & y-y_{W P, i} \quad h-h_{W P, i}
\end{array}\right] M_{i}\left[\begin{array}{ll}
x-x_{W P, i} & y-y_{W P, i} \quad h-h_{W P, i}
\end{array}\right]^{\mathrm{T}} \leq 1\right\},
$$

where $M_{i}$ is the shape matrix of a way point $W P_{i}$ centered at $x_{W P, i}, y_{W P, i}$, and $h_{W P, i}$. Likewise, the exclusion zones are defined as,

$$
E_{i}=\left\{(x, y, h): \frac{1}{2}\left[x-x_{E, i} y-y_{E, i} \quad h-h_{E, i}\right] P_{i}\left[x-x_{E, i} \quad y-y_{E, i} \quad h-h_{E, i}\right]^{\mathrm{T}} \leq 1\right\},
$$

where $P_{i}=\alpha I_{3}, \alpha>0$ and $I_{3}$ is the $3 \times 3$ Identity matrix.

This first simulation of this section adds an altitude requirement to the simulations above. It has a single way point centered at $(-0.3,0.3, .01)$ shaped as an ellipsoid with minor axes lengths of $0.1,0.1$, and 0.01 in the $x, y$, and $z$, directions. In addition, there are three large exclusion spheres with radii 0.01 centered at $(-0.2,-0.05, .01),(-0.7,-0.45, .01)$, and $(0.3,0.45, .01)$. The glider itself starts at a scaled altitude of 0.2 and restrictions are placed on the flight path angle such that it does not exceed $-0.0349 \mathrm{rad}$ and $0.0349 \mathrm{rad}$. The results are shown in Figs. 15-21.

It can be seen from Figs. 15-21 that the glider evades all of the exclusion zones while reaching the correct position and altitude of the way point. The vehicle at first ascends in order to reduce its velocity then easily travels to its destination. The trajectories of bank angle and flight path angle generated by the MPC controller are shown Fig. 16, and it can be seen that both angles obey the constraints. Note that the powered flight phases is initiated once $V$ reaches a value of 0.1 . The ECG also controls the longitudinal dynamics of the glider such that the vehicle reaches its desired flight path angle quickly. It is shown in Figs. 19-21 that the ECG control is done well within the flight constraints, as the lift, heating, angle-of-attack, elevator deflection, elevator deflection rate, and elastic deflection never exceed their limits.

The second simulation in this section involves multiple way points, with the first destination centered at $(-0.112,0.87, .03)$ and the second at $(0,2, .01)$, both shaped as ellipsoids with minor axes lengths of $0.1,0.1$, and 0.01 in the $x, y$, and $z$, directions. The exclusion spheres have radii 0.1 and are centered at $(0.17,1.3, .01)$ and $(-0.355,2.35, .01)$. The results are shown in Figs 22-28.

Once again, the glider is able to fly through each way point while avoiding the exclusion zones. The vehicle does not fly through the direct center of the first way point due to a number of factors. The first way point is placed fairly close to the glider's initial position, and its velocity is large enough that the control does not allow for such a maneuver. It appears that the glider flies as close to the center of the first way point as possible within control constraints. As in the previous simulation, the bank angle and flight path angle were optimized properly in order to reach the way points, with all control and structure constraints met. 


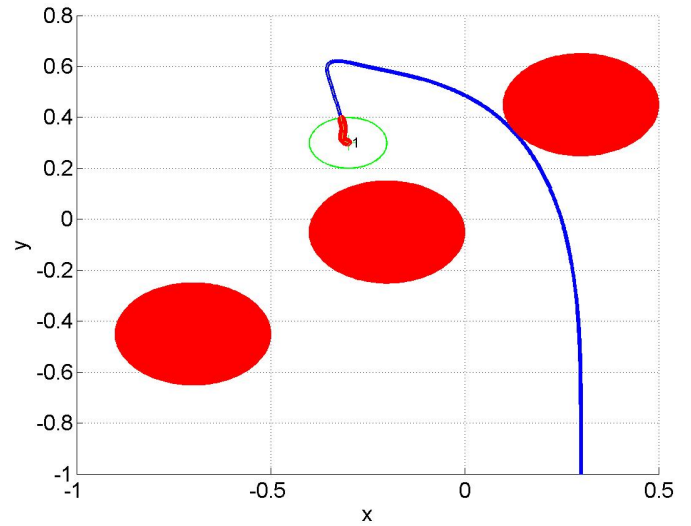

(a)
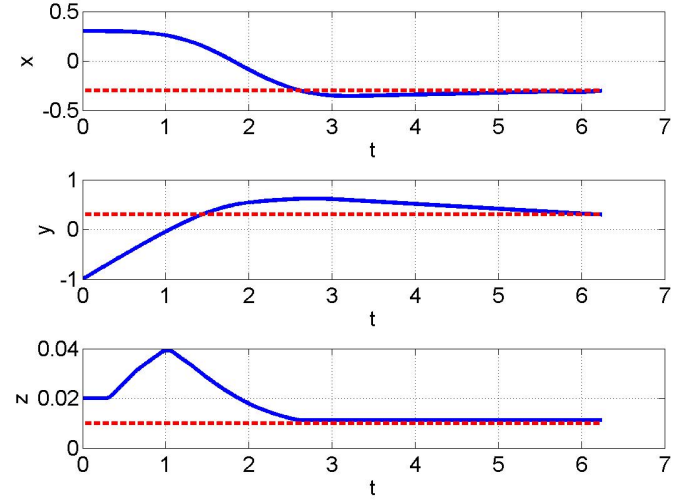

(b)

Figure 15. Left: Glider trajectory in the $x-y$ plane. Right: The time histories of $x, y$, and $z$. Dashed lines show the target positions as implied by the specified way points.

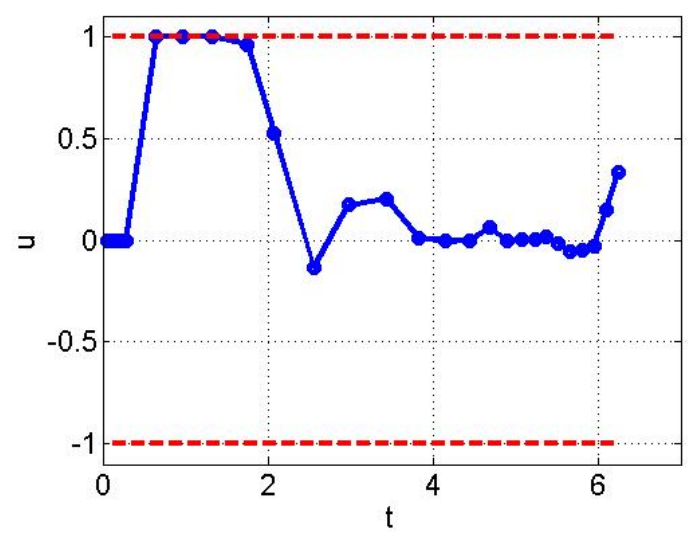

(a)

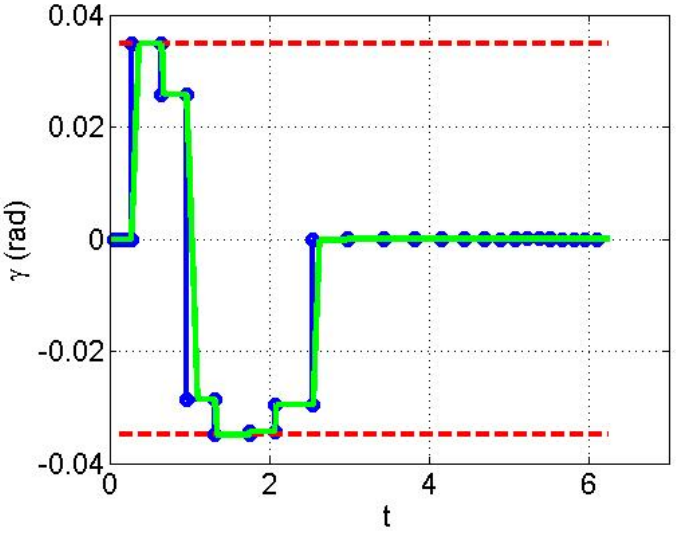

(b)

Figure 16. Left: Time history of normalized bank angle control signal. Note: bank angle sampled values are shown at the end of each control interval. Right: Time history of flight path angle control (blue) and actual flight path angle (green).

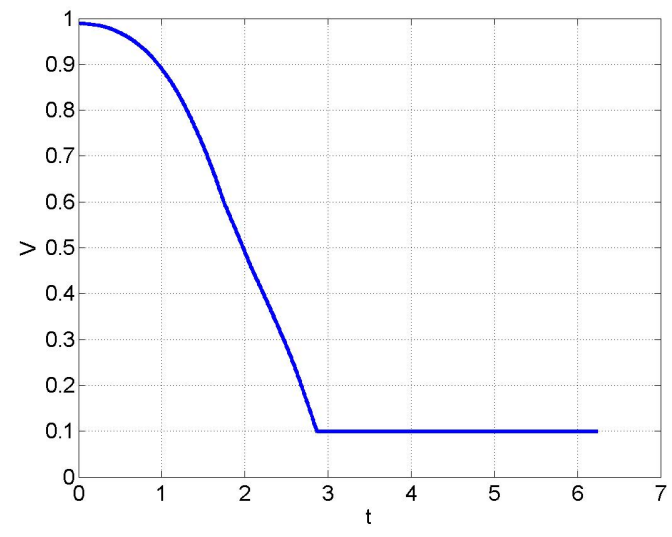

(a)

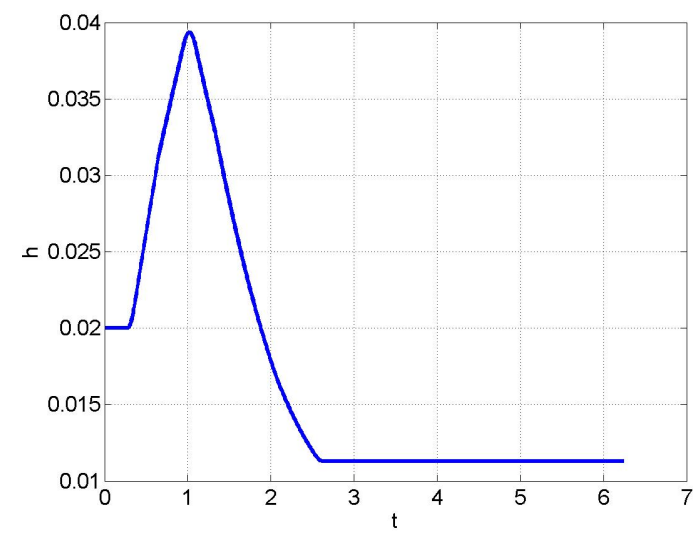

(b)

Figure 17. Left: Time history of glider velocity. Right: Time history of glider altitude. 


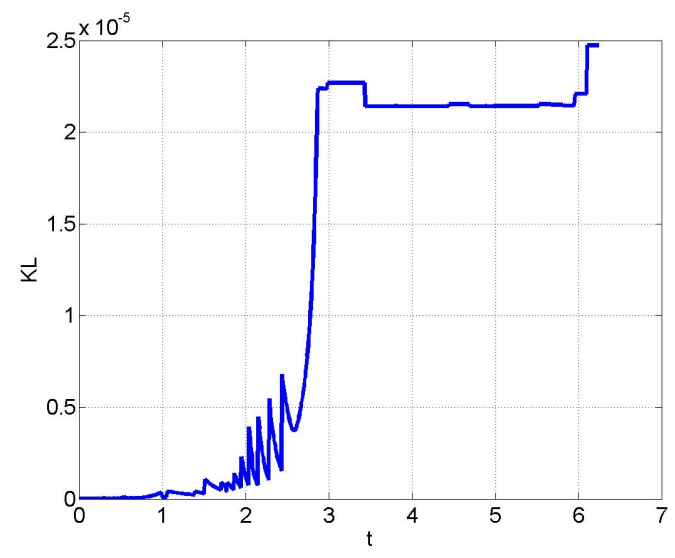

(a)

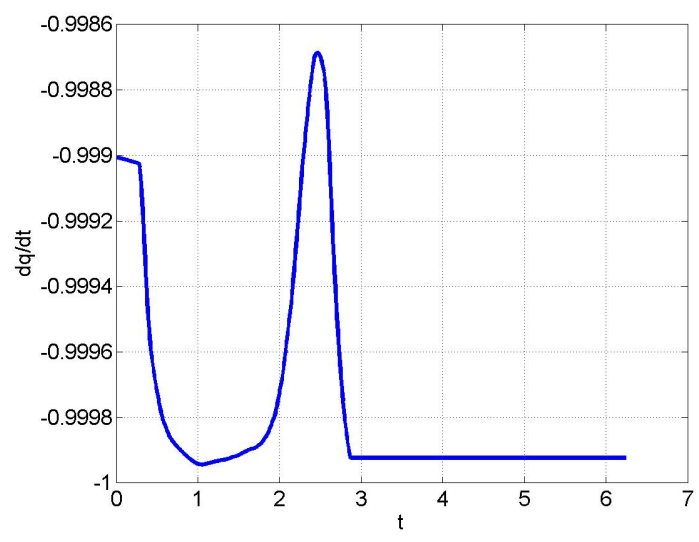

(b)

Figure 18. Left: Time history of glider lift coefficient $K_{L}$. Right: Time history of undimensional glider heating rate.

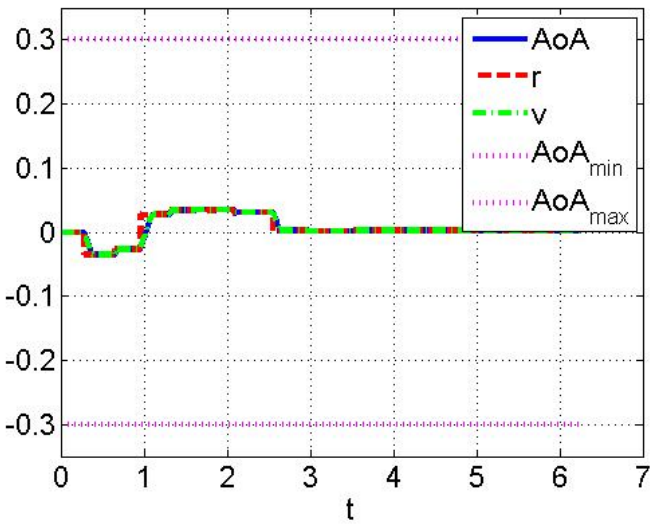

(a)

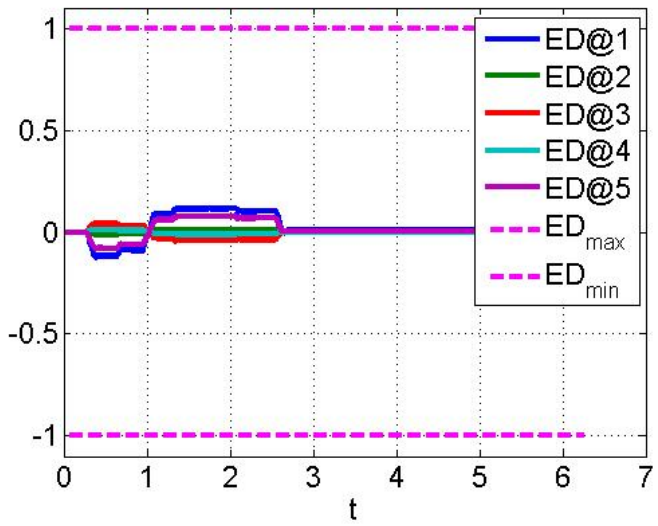

(b)

Figure 19. Left: The response of angle-of-attack (solid) with constraints. Right: The response of elastic deflection with constraints.

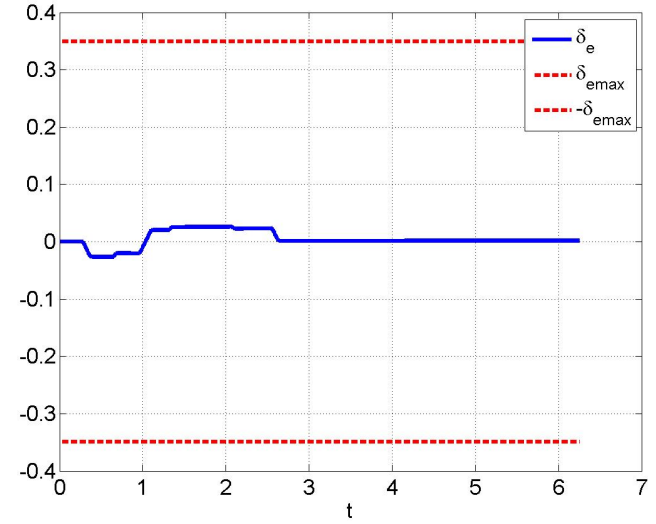

(a)

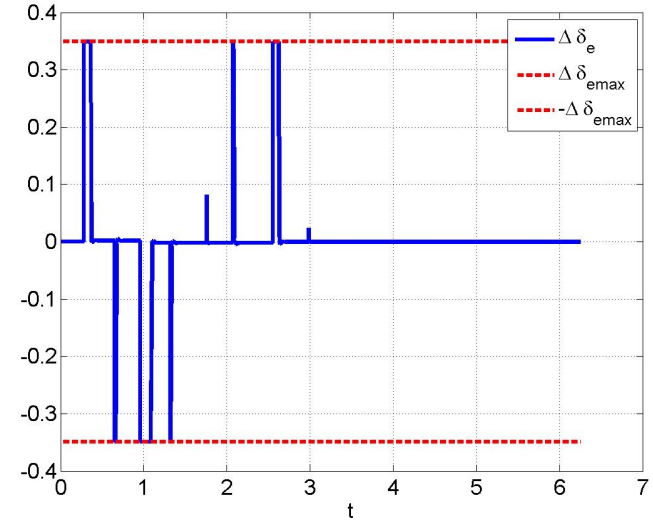

(b)

Figure 20. Left: The response of elevator deflection with constraints. Right: The response of elevator deflection with constraints. 


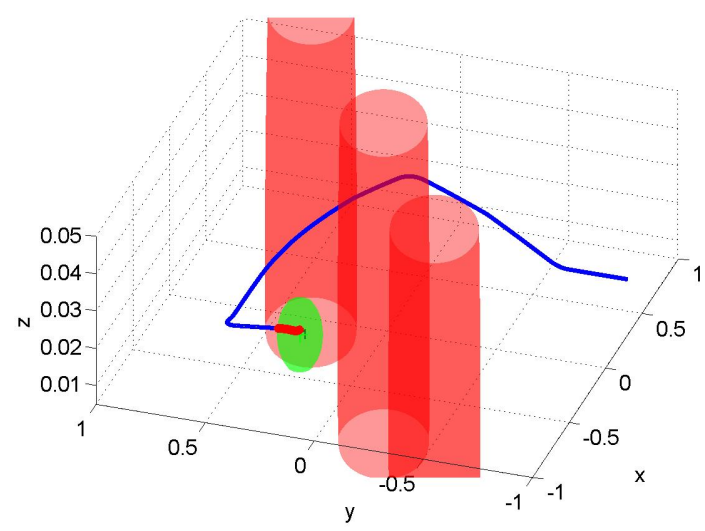

Figure 21. Glider trajectory in the $x-y-z$ frame.

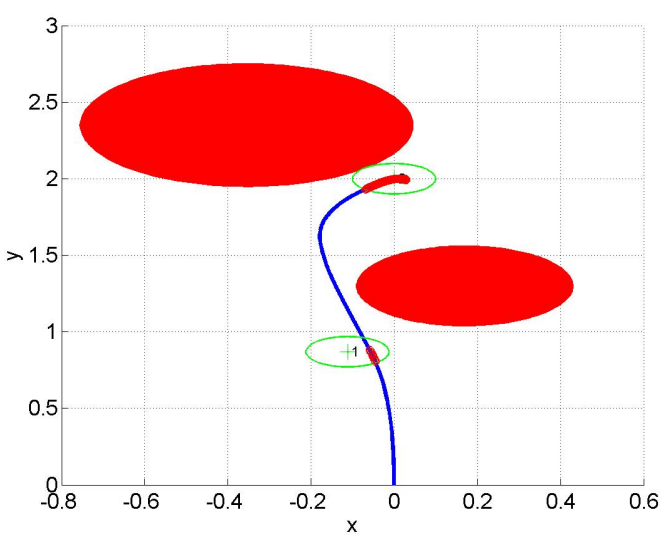

(a)
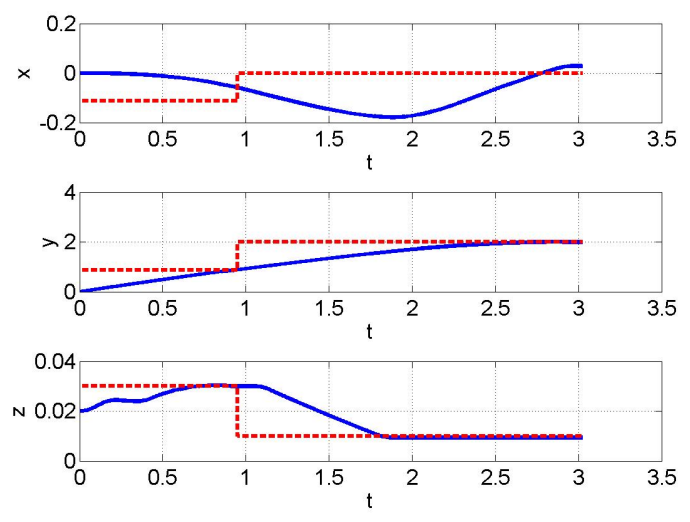

(b)

Figure 22. Left: Glider trajectory in the $x-y$ plane. Right: The time histories of $x, y$, and $z$. Dashed lines show the target positions as implied by the specified way points.

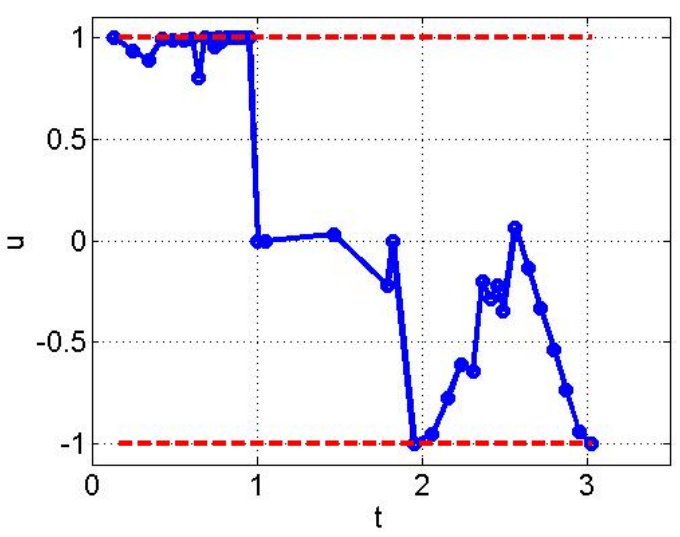

(a)

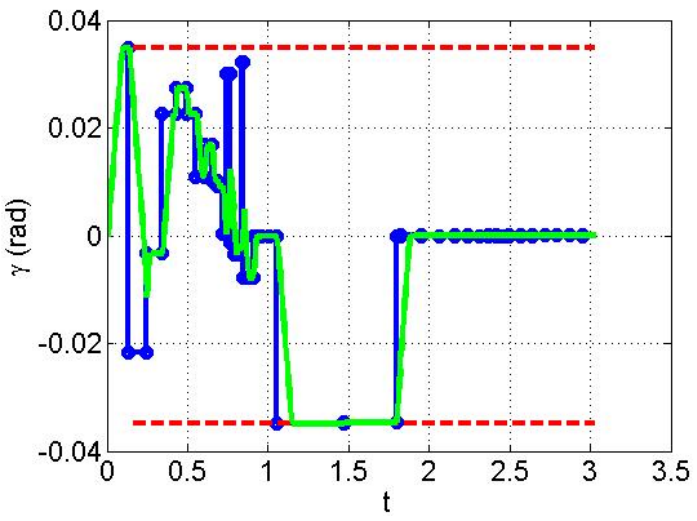

(b)

Figure 23. Left: Time history of normalized bank angle control signal. Note: bank angle sampled values are shown at the end of each control interval. Right: Time history of flight path angle control (blue) and actual flight path angle (green). 


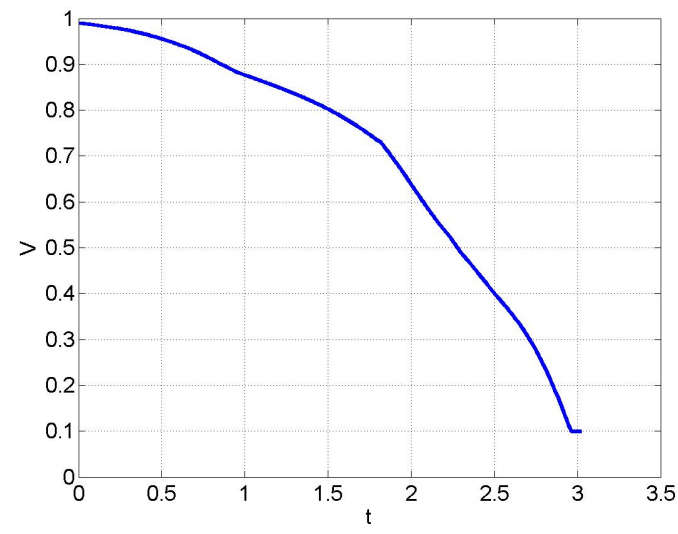

(a)

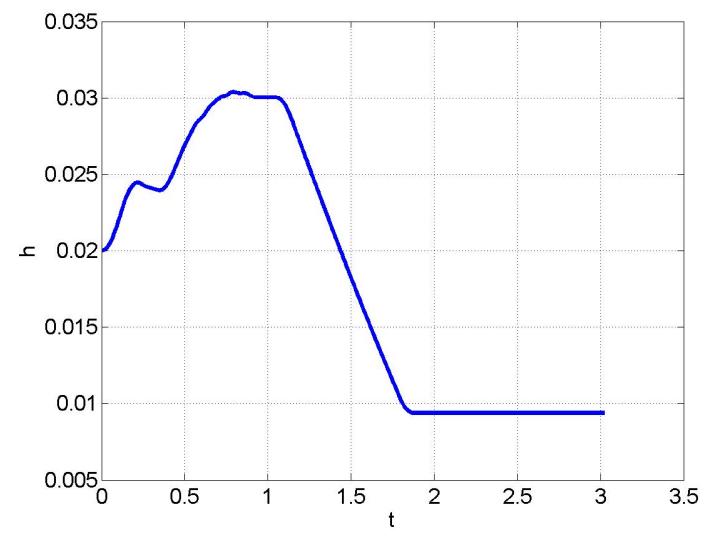

(b)

Figure 24. Left: Time history of glider velocity. Right: Time history of glider altitude.

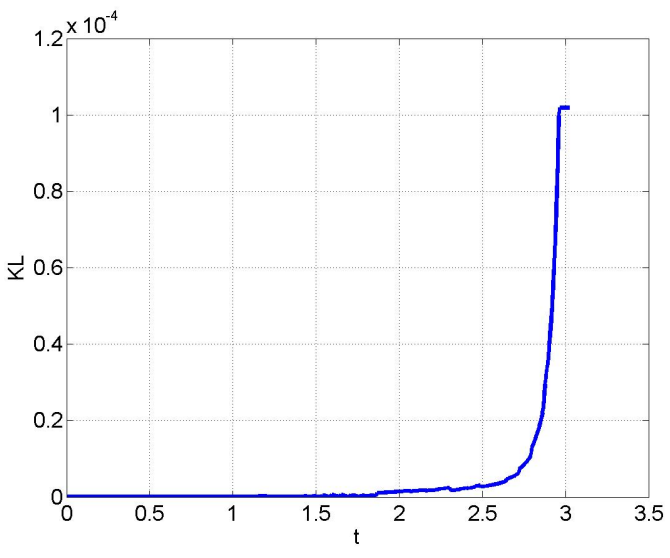

(a)

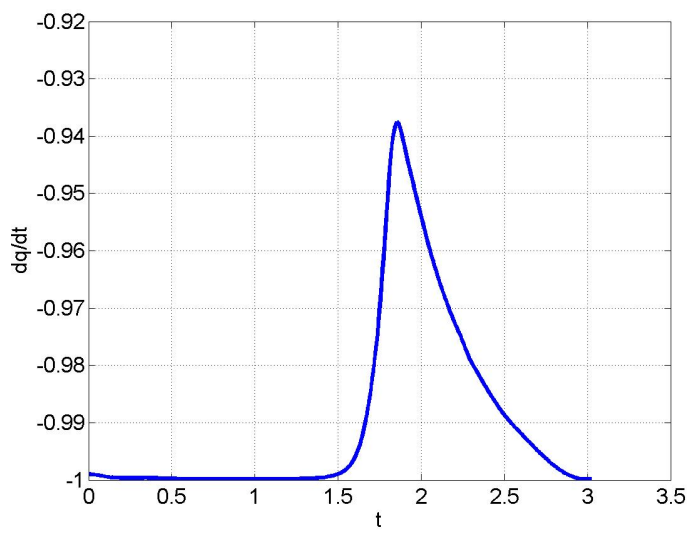

(b)

Figure 25. Left: Time history of glider lift coefficient $K_{L}$. Right: Time history of undimensional glider heating rate.

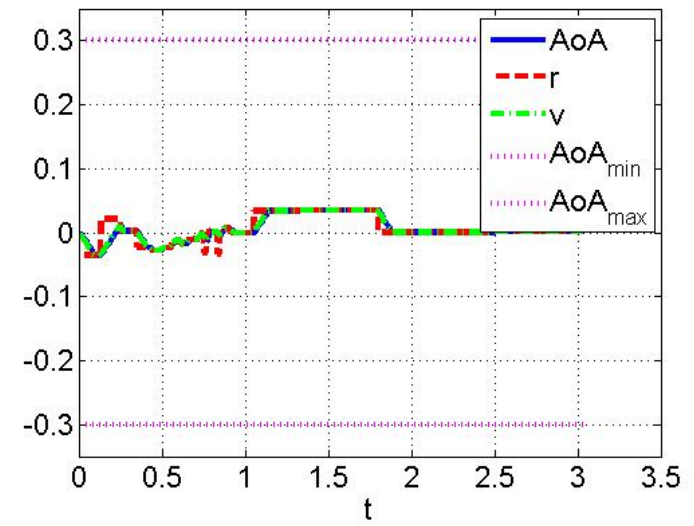

(a)

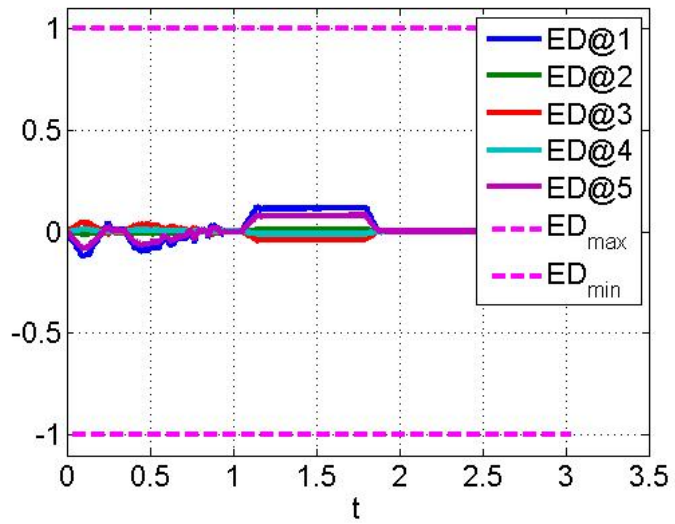

(b)

Figure 26. Left: The response of angle-of-attack (solid) with constraints. Right: The response of elastic deflection with constraints. 


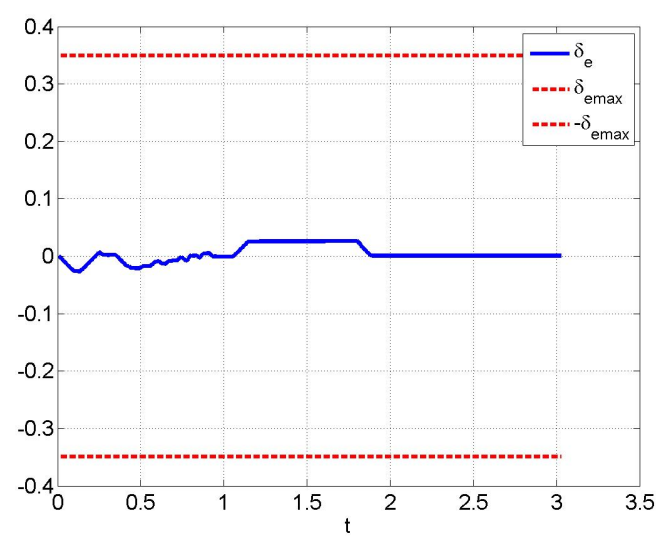

(a)

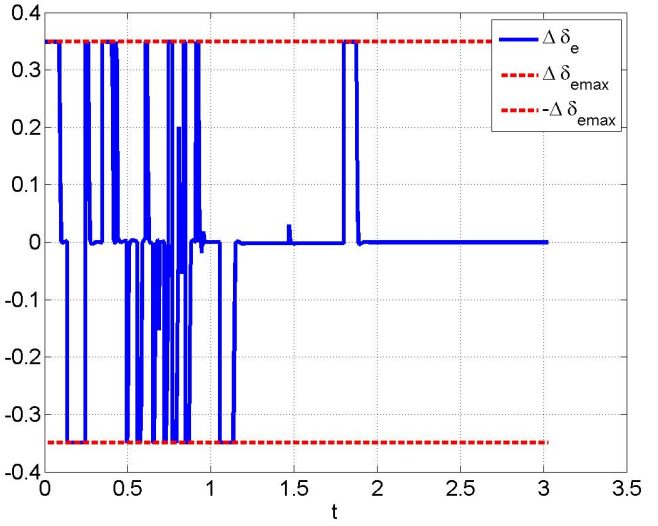

(b)

Figure 27. Left: The response of elevator deflection with constraints. Right: The response of elevator deflection rate with constraints.

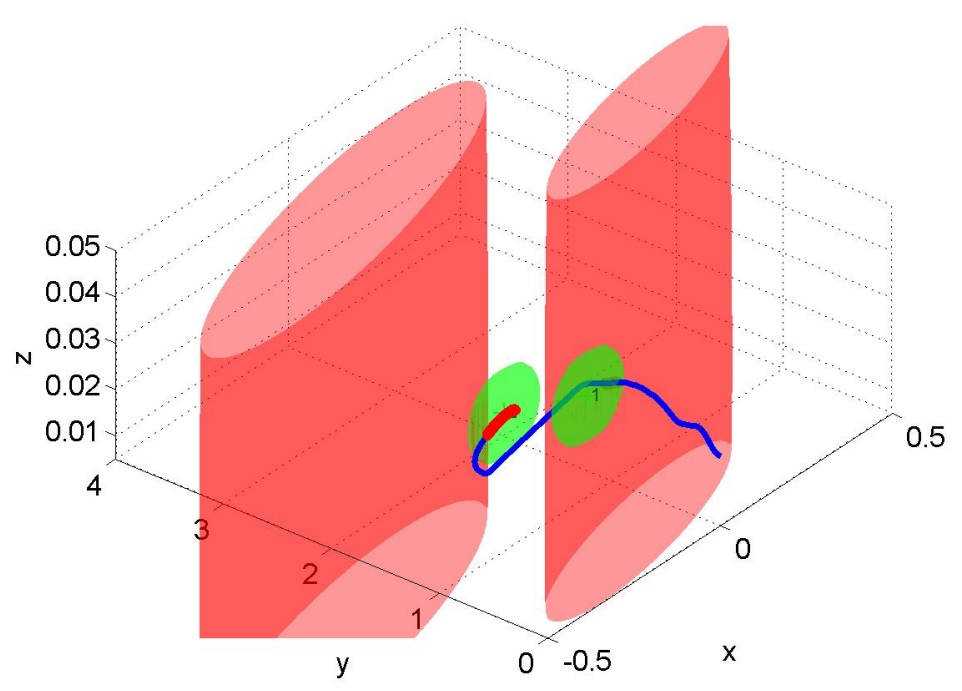

Figure 28. Glider trajectory in the $x-y-z$ frame. 


\section{Conclusion}

Hypersonic glider guidance and control involves numerous challenges, including, but not limited to, multiple control and structure constraints, disturbances/uncertainties, and changing environments. This paper proposes a combined Model Predictive Control outer-loop and a LQ-PI/ECG inner-loop for hypersonic vehicle control. Using MPC as the outer-loop provides a capability for real-time adjusting of the flight trajectory in response to the appearance/disappearance of exclusion zones, disturbances, and failure models. The LQ-PI/ECG inner-loop ensures tracking of the outer-loop generated set-points subject to satisfying the inner-loop constraints. Future research will include implementing this control architecture with special on-board, real-time optimization algorithms while inducing disturbances and time varying exclusion zones to demonstrate robustness.

\section{Acknowledgements}

The authors thank Dr. Michael Bolander of AFRL for providing the model used in Section III and Uros Kalabic of University of Michigan for providing ECG design software.

\section{References}

\footnotetext{
${ }^{1}$ Mali, T., "Superfast military aircraft lost in test flight," http://www.space.com/12607-darpa-launches-hypersonic-glidermach-20-test-flight.html, 2011.

${ }^{2}$ Chen, H., Ning, L., and Shaoyuan, L., "Switching multi-model predictive control for hypersonic vehicle," Proceedings of 8th Asian Control Conference (ASCC) 2011, 2011.

${ }^{3}$ Soloway, D., Rodriguez, A., Dickeson, J., Cifdaloz, O., Benavides, J., Sridharan, S., Kelkar, A., and Vogel, J., "Constraint enforcement for scramjet-powered hypersonic vehicles with significant aero-elastic-propulsion interactions," Proceedings of American Control Conference 2009, 2009.

${ }^{4}$ Weiwei, Q., Zhiqiang, Z., Li, Z., and Gang, L., "Robust Model Predictive Control for hypersonic vehicle based on LPV," Proceedings of the IEEE International Conference on Information and Automation 2010, 2010.

${ }^{5}$ Tao, X., Hua, C., Li, N., and Li, S., "Robust Model Predictive Controller design for a hypersonic flight vehicle," Proceedings of International Conference on Modelling, Identification and Control 2012, 2012.

${ }^{6}$ Zinnecker, A., Serrani, A., Bolender, M., and Doman, D., "Combined reference governor and anti-windup design for constrained hypersonic vehicles models," August 2009.

${ }^{7}$ Kahveci, N. and Kolmanovsky, I., "Constrained control of UAVs using adaptive anti-windup compensation and reference governors," Proceedings of SAE AeroTech Congress and Exhibition 2009, 2009.

${ }^{8}$ Starek, J. and Kolmanovsky, I., "Nonlinear Model Predictive Control strategy for low thrust spacecraft missions," Optimal Control, Applications and Methods, Accepted for publication.

${ }^{9}$ Baldwin, M. and Kolmanovsky, I., "Hypersonic glider guidance using Model Predictive Control," Proceedings of American Control Conference 2013, 2012.

${ }^{10}$ Baldwin, M. and Kolmanovsky, I., "Constrained inner-loop control of a hypersonic glider using extended command governor," Proceedings of American Control Conference 2013, 2012.

${ }^{11}$ Jorris, T., "Common aero vehicle autonomous reentry trajectory optimization satisfying waypoint and no-fly zone constraints," Ph.D. dissertation, Air Force Institute of Technology, 2007.

${ }^{12}$ Wiesel, W., Spaceflight Mechanics, 3rd Edition, Aphelion Press, 2010.

${ }^{13}$ Gilbert, E. and Ong, C., "An Extended Command Governor for Constrained Linear Systems with Disturbances," Proceedings of Joint 48th IEEE Conference on Decision and Control and 28th Chinese Control Conference 2009.

${ }^{14}$ Bolender, M., "An overview on dynamics and controls modelling of hypersonic vehicles," Proceedings of American Control Conference 2009, 2009.

${ }^{15}$ Kolmanovsky, I., Kalabic, U., and Gilbert, E., "Developments in constrained control using reference governors," Proceedings of the IFAC Conference on Nonlinear Model Predictive Control (NMPC) 2012, 2012, pp. 282-290.

${ }^{16}$ Gilbert, E. and Ong, C., "Constrained linear systems with hard constraints and disturbances: An extended command governor with large domain of attraction," Automatica, Vol. 47, 2011, pp. 334-340.

${ }^{17}$ Bemporad, A., Morari, M., Dua, V., and Pistikopoulos, "The explicit solution of Model Predictive Control via multiparametric quadratic programming," Automatica, Vol. 38, No. 3, 2002.

${ }^{18}$ Kalabic, U., Kolmanovsky, I., Buckland, J., and Gilbert, E., "Reference and extended command governors for control of turbocharged gasoline engines based on linear models," Proceedings of IEEE Multi-Conference on Systems and Control 2011.

${ }^{19}$ Kalabic, U., Gilbert, E., and Kolmanovsky, I., "Reduced order reference governor," Proceedings of 51st IEEE Conference Decision and Control, 2012.
} 